MZ-TH/05-17

hep-ph/0508173

August 2005

\title{
Evaluating massive planar two-loop tensor vertex integrals
}

\author{
Stefan Groote ${ }^{1,2}$ and Markus M. Knodel ${ }^{1}$ \\ ${ }^{1}$ Institut für Physik der Johannes-Gutenberg-Universität, \\ Staudinger Weg 7, 55099 Mainz, Germany \\ ${ }^{2}$ Füüsika-Keemiateaduskond, Tartu Ülikool, Tähe 4, 51010 Tartu, Estonia
}

\begin{abstract}
Using the parallel/orthogonal space method, we calculate the planar two-loop three-point diagram and two rotated reduced planar two-loop three-point diagrams. Together with the crossed topology, these diagrams are the most complicated ones in the two-loop corrections necessary, for instance, for the decay of the $Z^{0}$ boson. Instead of calculating particular decay processes, we present the new algorithm which allows one to calculate arbitrary NNLO calculations for massive planar two-loop vertex functions in the general mass case. All integration steps up to the last two ones are performed analytically and will be implemented under xloops as part of the Mainz xloops-GiNaC project. The last two integrations are done numerically using methods like VEGAS and Divonne. Thresholds originating from Landau singularities are found and discussed in detail. In order to demonstrate the numeric stability of our methods we consider particular Feynman integrals which contribute to different physical processes. Our results can be generalized to the case of the crossed topology.
\end{abstract}




\section{Introduction}

Precision measurements at the LEP collider at CERN and other colliders like e.g. SLC at SLAC, TEVATRON at Fermilab and HERA at DESY have reached a precision which has exceeded all expectations. This is true especially for electron colliders [1, 2, 3, 4]. At the moment the precision of measurements related to the parameters of the Standard Model of electroweak interactions reaches values up to $O\left(10^{-4}\right)$ [4. At future colliders like the LHC or the ILC (including GigaZ), further improvements are expected [3, 5, 6, 7, 8, .

Compared to this, theoretical predictions accomplish this precision only in very few cases. To check the validity of the Standard Model and to be able to draw conclusions about "new physics", progress in theoretical methods and their application is necessary [2, 3]. The complexity of the calculations and the number of the graphs which have to be calculated within perturbation theory grows considerably order by order. At second order only a few observables are calculated [9]. Still there is no method available which allows for the (semi)automatic calculation of arbitrary processes at this order. Much work has been done on NNLO calculations [10, 11, 12, 13, 14, 15, 16, 17, 18, 19, 20, 21, 22, 23, 24, 25, 26, 27]. While for mixing QCD and electroweak $O\left(\alpha \alpha_{s}\right)$ NNLO vertex corrections a few calculations were done several years ago [17], for the evaluation of two-loop threepoint diagrams in the general mass case only the methods proposed in Refs. [28, 29, 30] have been used successfully in order to calculate electroweak NNLO corrections [31]. With increasing energy as it will be used at the ILC, radiative corrections will become increasingly important. Therefore, there is still need for independent methods to calculate general massive two-loop vertex diagrams.

In this paper we present a new algorithm which allows one to calculate NNLO-corrections for general massive planar two-loop vertex functions as they arise in the Standard Model of electroweak interactions using Feynman gauge, e.g. for the effective weak mixing angle $\sin ^{2} \theta_{\text {eff }}^{\text {lept }}$ [31, 32] occuring in $Z^{0} \rightarrow l^{+} l^{-}$and for other processes like $Z^{*} \rightarrow t \bar{t}$. Even though the calculation of particular physical processes is not subject of this paper, the new algorithm enables one to perform such calculations. For our algorithm we use the parallel/orthogonal space method [33] which allows one to separate effects coming from inner momenta from those of momenta of the outer particles of the process.

The benefit of the parallel/orthogonal space method is the fact that the calculation stays close to the physical process. The introduction of Feynman parameters and the ap- 
plication of the Wick rotation which estrange the calculation from the physical process for other methods are not necessary for the parallel/orthogonal space method. Instead, a decay process can be calculated in the rest frame of the decaying particle. The introduction of Gram determinants which might cause artificial divergences is not necessary. The analytical integration leads to still rather simple basic functions like logarithms and dilogarithms. For the remaining numerical integration the integrand can be analyzed at physical thresholds. Landau singularities are mirrored directly onto the parameters of the integrands. Finally, the method is totally independent from other methods and therefore allows for an independent check.

Up to now, the parallel/orthogonal space method was used successfully to evaluate the general massive scalar integrals in the case of the planar topology [34] and in the case of the crossed topology [35. However, it was not possible to evaluate two-loop three-point functions which contain loop momenta in the numerator. The method proposed in this paper allows one to calculate also tensor integrals for the planar vertex topologies.

We present a tensor reduction which reduces the planar and rotated reduced planar two-loop three-point topologies containing an arbitrary tensor structure to a set of master integrals with strongly restricted numerator structure [36]. As for most of the master integrals which remain after tensor reduction there are already established methods available [24, 25, 26, 27], we do not give results in these cases. The same is valid for particular mass cases [10, 37, 38, 39] and for the UV-singular parts [36]. Efficient methods for several cases including few masses have been developed in Refs. [19, 20, 21]. We also do not dwell on the calculation of massless diagrams for which other methods are applied successfully (see e.g. [40]). As IR divergences occur in this case, there is no systematic method available within the parallel/orthogonal space method because for each special case the subtraction has to be found seperately "by hand" 41, 42. Instead, we concentrate on the most complicated part, namely the calculation of the UV-finite parts of those master integrals which keep the planar or rotated reduced planar topology after tensor reduction.

Two-loop integration in four-dimensional space-time needs eight integration steps. Using the parallel/orthogonal space method, we can do six of them analytically using different techniques. The last two integration steps are done numerically. Because most of the integrations can be performed analytically, the Landau singularities of the Feynman diagrams can be related graphically to the integrands in the remaining two-dimensional integration region. Using different algorithms, we demonstrate the numerical stability. 
The algorithm for the numerical calculation of the last two integrations is developed and its reliability is demonstrated. In addition, further exhaustive numerical tests are done. While for the numerical integration programs like the Monte Carlo integration routine VEGAS [43, 44] and the program Divonne of the library CUBA [45] are used, the implementation of the algorithms for the semi-automatical calculation in xloops [46, 47, 48] is part of the work described in this paper. In the xloops-GiNaC project developed by the members of the ThEP working group in Mainz [49, 50, work on the automatic generation of Feynman diagrams and their evaluation with analytical and numerical means is in progress. For this purpose the language for algebraic calculation $\mathrm{GiNaC}$ was developed [51. While most of the master two-loop integrals not considered in this paper are already implemented in $x$ loops, the missing planar topologies will be added in the course of the work introduced here. The complete set of algorithms described in this paper is implemented under xloopsGiNaC. However, the implementation of parts of the algorithms for known topologies as mentioned in Sec. 3.8 still has to be done. Because in this spirit the implementation is not yet finished, the calculation of physical processes is not subject of this paper but will be presented in a future publication.

The paper is organized as follows. In Sec. 2 we introduce the main tools of the analytical calculation associated with the parallel/orthogonal space method. The tensor reduction procedure is explained in detail in Sec. 3 for the non-reduced planar two-loop three-point diagram, and modifications in case of the rotated reduced planar diagrams are mentioned and explained. The tensor reduction leads to master integrals which are integrated analytically in Sec. 4 up to the last two integrations. In Sec. 5 we deal with the analysis of Landau singularities and thresholds. Sec. 6 is devoted to the numerical integration. In both sections we present examples to show the reliability of our procedure. In Sec. 7 we give our conclusions. In Appendix A we deal with the integral basis.

\section{Tools for the calculation}

In this section we provide the reader with the tools necessary for the calculation of massive two-loop tensor vertex integrals. Most of the tools were already introduced in the literature [33, 34, 35, 42, 52, 53, 54]. Therefore, we can be brief in presenting these tools. 


\subsection{The parallel/orthogonal space method}

The integrals we have to calculate are determined by the two momenta of the produced particles, $p_{1}$ and $p_{2}$. Because the integrals are expressed in terms of covariant quantities, we are free to decompose any loop momentum $k$ into two covariant vectors, $k^{\mu}=k_{\|}^{\mu}+k_{\perp}^{\mu}$, where $k_{\|}$has components in the parallel space which is the linear span of the external momenta $p_{i}$, while $k_{\perp}$ is the orthogonal complement with $\sum_{\mu} k_{\perp}^{\mu} p_{i \mu}=0$ with components in the orthogonal space [10, 17]. For three-point functions we can consider the process in the rest frame of the decaying particle. In this frame the two emerging particles are produced back-to-back, defining the $z$-axis. ${ }^{1}$ In this frame the representation

$$
p_{1}=\left(E_{1} ; q_{z}, 0,0\right), \quad p_{2}=\left(E_{2} ;-q_{z}, 0,0\right), \quad p=p_{1}+p_{2}=(E ; 0,0,0)
$$

can be used where $p$ is the momentum of the decaying particle with $E=E_{1}+E_{2}$ and $p^{2}=E^{2}$. Accordingly, the loop momenta $k$ and $l$ necessary for the description of the two-loop integral are parametrized by 53

$$
k=\left(k_{0} ; k_{1}, \vec{k}_{\perp}\right), \quad l=\left(l_{0} ; l_{1}, \vec{l}_{\perp}\right) .
$$

The two-dimensional vectors $\vec{k}_{\perp}$ and $\vec{l}_{\perp}$ are represented in polar coordinates, using the squared absolute values $s=\vec{k}_{\perp}^{2}=k_{\perp}^{2}$ and $t=\vec{l}_{\perp}^{2}=l_{\perp}^{2}$ and two angles, the angle $\alpha$ of $\vec{k}_{\perp}$ with the $x$-axis and the relative angle $\gamma$. We can write

$$
\vec{k}_{\perp} \cdot \vec{l}_{\perp}=k_{\perp} l_{\perp} z=\sqrt{s t} z, \quad z=\cos \gamma=\cos \left(\vec{k}_{\perp}, \vec{l}_{\perp}\right)
$$

The choice of parallel and orthogonal subspaces itself is Lorentz invariant, so that the calculation can still be done in any Lorentz frame. The benefit of the parallel/orthogonal space method ( $\mathrm{P} / \mathrm{O}$-space method) is that the contributions belonging to the orthogonal space can be integrated out and we are left with the contributions in parallel space only. The integration measure is accordingly written as

$$
\int d^{4} k \int d^{4} l=\pi \int_{-\infty}^{+\infty} d k_{0} d l_{0} d k_{1} d l_{1} \int_{0}^{\infty} d s d t \int_{-1}^{+1} \frac{d z}{\sqrt{1-z^{2}}}
$$

where the trivial integration over $\alpha$ has already been performed.

\footnotetext{
${ }^{1}$ The $z$-component will be written as the second component of the four-vector in the following.
} 


\subsection{The linearization}

Using Feynman gauge, the denominators of integrals occurring in two-loop vertex calculations contain up to six propagator factors. A typical factor is given by

$$
P_{1}=\left(k+p_{1}\right)^{2}-m_{1}^{2}+i \eta
$$

where $\eta>0$. In the $\mathrm{P} / \mathrm{O}$-space representation this propagator factor reads

$$
P_{1}=\left(k_{0}+E_{1}\right)^{2}-\left(k_{1}+q_{z}\right)^{2}-k_{\perp}^{2}-m_{1}^{2}+i \eta
$$

If we replace $k_{0}=k_{0}^{\prime} \pm k_{1}$, we obtain

$$
P_{1}=\left(k_{0}^{\prime}+E_{1}\right)^{2} \pm 2 k_{1}\left(k_{0}^{\prime}+E_{1} \mp q_{z}\right)-q_{z}^{2}-k_{\perp}^{2}-m_{1}^{2}+i \eta
$$

where $k_{1}$ no longer appears quadratically. This replacement, also known as linearization, is allowed because the integral for $k_{0}$ ranges from $-\infty$ to $+\infty$. However, because of the occurrence of the mixing propagator factor

$$
P_{3}=(k+l)^{2}-m_{3}^{2}+i \eta
$$

the signs for the linearizations in $k$ and $l$ are coupled. In applications of this linearization we use the sign which is the most appropriate for our aims. The benefit of the linearization is that the integrals over $k_{1}$ and $l_{1}$ can then be calculated by using the residue theorem.

\subsection{The integration over $z$}

The quantity $z$ occurs only in the just mentioned propagator factor $P_{3}$. After linearization this propagator factor can be written as $P_{3}=A+B z+i \eta$ where

$$
A=A\left(k_{1}, l_{1}\right)=\left(k_{0}^{\prime}+l_{0}^{\prime}\right)^{2} \pm 2\left(k_{1}+l_{1}\right)\left(k_{0}^{\prime}+l_{0}^{\prime}\right)-(s+t)-m_{3}^{2}, \quad B=-2 \sqrt{s t}
$$

$P_{3}$ occurs only once in the denominator (if at all). The resulting integral

$$
\int_{-1}^{+1} \frac{d z}{\sqrt{1-z^{2}}} \frac{1}{A+B z+i \eta}=\frac{\pi}{\sqrt[\star]{(A+i \eta)^{2}-B^{2}}}=\frac{\pi}{\sqrt[\star]{(A+i \eta)^{2}-4 s t}}
$$

can be written in this closed form only if we make a convention different from the usual one [10]. We stipulate that the cut of the square root is located on the positive real axis

instead of the negative one. For this reason we have added the star in $\sqrt[\star]{(A+i \eta)^{2}-B^{2}}$ 
which reminds one of the different cut. The occurrence of the modified square root has consequences for the subsequent integrations because in closing paths for the residue theorem we have to avoid crossing this cut. In order to find conditions for this we split the square root up into a product of two square roots $\sqrt[\star]{A \pm B+i \eta}$. If one of the radicands is equal to a positive real number $x$, we are definitely crossing the cut. Written in terms of $k_{1}$ and $l_{1}$, we obtain

$$
\left(k_{1}+l_{1}\right)^{\mathrm{cut}}= \pm \frac{x-\left(k_{0}^{\prime}+l_{0}^{\prime}\right)^{2}+m_{3}^{2}+(\sqrt{s} \mp \sqrt{t})^{2}-i \eta}{2\left(k_{0}^{\prime}+l_{0}^{\prime}\right)} .
$$

Note that the two undetermined signs are not correlated. While the sign between $\sqrt{s}$ and $\sqrt{t}$ depends on which of the two square roots is taken, the global sign of the right hand side is due to the sign of the linearization. Considering only the linearizations $k_{0} \rightarrow k_{0}^{\prime}+k_{1}$,

$l_{0} \rightarrow l_{0}^{\prime}+l_{1}$, the signs of the real and imaginary part of the cut still depend on the sign of $\left(k_{0}^{\prime}+l_{0}^{\prime}\right)$. If $\left(k_{0}^{\prime}+l_{0}^{\prime}\right)>0$, the cut is located in the lower complex half plane. The real part starts (for $x=0$ ) at some finite value and runs to $+\infty$, independent of which square root we selected. In order to avoid the cut, we therefore have to close the integration path for $k_{1}$ resp. $l_{1}$ in the upper complex half plane while for $\left(k_{0}^{\prime}+l_{0}^{\prime}\right)<0$ we have to close it in the lower half plane. The situation is opposite for the linearization with a minus sign.

\section{Tensor reduction}

After having introduced the main tools for the calculation, we can start with the calculation itself. The starting point is the tensor integral

$$
\mathcal{T}_{a_{0} a_{1} a_{2} b_{0} b_{1} b_{2} c}^{0}=\int \frac{k_{0}^{a_{0}} k_{1}^{a_{1}}\left(k_{\perp}^{2}\right)^{a_{2}} l_{0}^{b_{0}} l_{1}^{b_{1}}\left(l_{\perp}^{2}\right)^{b_{2}}\left(k_{\perp} l_{\perp} z\right)^{c}}{P_{1} P_{2} P_{3} P_{4} P_{5} P_{6}} d^{4} k d^{4} l
$$

where

$$
\begin{aligned}
P_{1}=\left(k+p_{1}\right)^{2}-m_{1}^{2}+i \eta & P_{4}=\left(l-p_{1}\right)^{2}-m_{4}^{2}+i \eta \\
P_{2}=\left(k-p_{2}\right)^{2}-m_{2}^{2}+i \eta & P_{5}=\left(l+p_{2}\right)^{2}-m_{5}^{2}+i \eta \\
P_{3}=(k+l)^{2}-m_{3}^{2}+i \eta & P_{6}=l^{2}-m_{6}^{2}+i \eta .
\end{aligned}
$$

If one is doing Standard Model calculations using Feynman gauge, the powers are restricted by $0 \leq a_{0}, a_{1}, 2 a_{2} \leq 3$ and $0 \leq b_{0}, b_{1}, 2 b_{2}, c \leq 4$, as well as by $0 \leq a_{0}+a_{1}+2 a_{2}+c \leq 3$ and $1 \leq b_{0}+b_{1}+2 b_{2}+c \leq 4$. Nevertheless, the algorithm introduced in this paper works for 


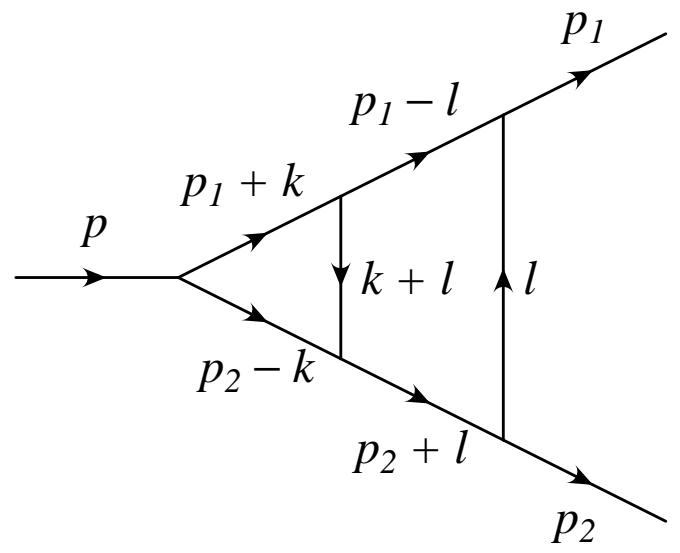

Figure 1: Momentum flow convention for the planar two-loop three-point function

general powers. In Fig. 11 we show the momentum flow for the diagram in order to define the momenta. For the indices $a_{i}, b_{i}, c$ we allow non-negative integer values. In performing the tensor reduction the integrals are simplified to integrals with simpler numerator and/or denominator structure.

For one-loop integrals the numerator can always be removed by reduction procedures [55]. For two-loop integrals this need not be the case. In general there are not enough propagator factors to cancel all components of the numerator that occur. For the genuine planar two-loop three-point function all numerator factors related to the second loop momentum $l$ can be cancelled. This is not the case for the reduced topologies, and it is not the case for the first loop momentum $k$. The aim of the tensor reduction in general is to reduce the numerator as far as possible so that the integrations can be performed similar to what is done for a trivial numerator [34]. The reduction procedure will be explained in the following. The representation of the procedure by diagrams will show the topologies only. In this spirit plus signs like the one occuring in Eq. (19) have to be understood as sums over diagrams with the same topology but different factors and signs.

\subsection{Cancellation of the mixed contribution}

The first step in the cancellation procedure consist in cancelling powers of the mixed factor $\left(k_{\perp} l_{\perp} z\right)$. This factor occurs also in the propagator factor $P_{3}$. We can write

$$
2 k_{\perp} l_{\perp} z=N_{3}-P_{3}
$$


where

$$
N_{3}=k_{0}^{2}-k_{1}^{2}-k_{\perp}^{2}+l_{0}^{2}-l_{1}^{2}-l_{\perp}^{2}+2 k_{0} l_{0}-2 k_{1} l_{1}-m_{3}^{2}+i \eta .
$$

Used iteratively, we obtain

$$
\left(k_{\perp} l_{\perp} z\right)^{c}=\left(\frac{N_{3}}{2}\right)^{c}-\sum_{i=0}^{c-1} \frac{N_{3}^{i}}{2^{i+1}}\left(k_{\perp} l_{\perp} z\right)^{c-i-1} P_{3} .
$$

This iterative formula is necessary because the propagator factor $P_{3}$ occurs only once. As applied to the integral, we obtain

$$
\int \frac{\left(k_{\perp} l_{\perp} z\right)^{c} d^{4} k d^{4} l}{P_{1} P_{2} P_{3} P_{4} P_{5} P_{6}}=\frac{1}{2^{c}} \int \frac{N_{3}^{c} d^{4} k d^{4} l}{P_{1} P_{2} P_{3} P_{4} P_{5} P_{6}}-\sum_{i=0}^{c-1} \frac{1}{2^{i+1}} \int \frac{N_{3}^{i}\left(k_{\perp} l_{\perp} z\right)^{c-i-1}}{P_{1} P_{2} P_{4} P_{5} P_{6}} d^{4} k d^{4} l
$$

where we have skipped all the other numerator factors for convenience. The first part is the same planar integral with a different numerator structure. However, the second part no longer contains the mixing propagator factor $P_{3}$. Instead, the diagram factorizes. In addition, we can conclude that this vanishes if $c-i-1$ is odd. The reason is that the integration over $z$ is given by

$$
\int_{-1}^{+1} \frac{z^{c-i-1} d z}{\sqrt{1-z^{2}}}
$$

which vanishes if the integrand is odd. Diagramatically we can write

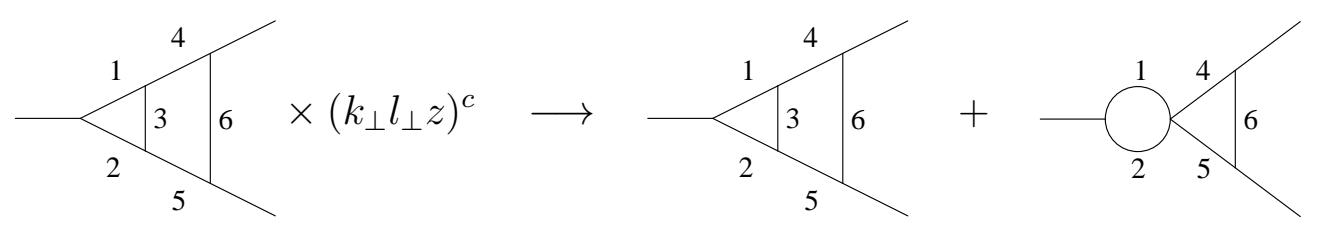

After having cancelled the mixing part it is irrelevant with which loop momentum we continue. However, we always start with the orthogonal space components.

\subsection{Cancellation of $k_{\perp}^{2}$}

The factor $k_{\perp}^{2}=s$ occurs in the denominator factor $P_{1}$. We can write

$$
k_{\perp}^{2}=N_{1}-P_{1}, \quad N_{1}=k_{0}^{2}-k_{1}^{2}+2 k_{0} E_{1}-2 k_{1} q_{z}+E_{1}^{2}-q_{z}^{2}-m_{1}^{2}+i \eta
$$

The iterative formula

$$
\left(k_{\perp}^{2}\right)^{a_{2}}=N_{1}^{a_{2}}-\sum_{i=0}^{a_{2}-1} N_{1}^{i}\left(k_{\perp}^{2}\right)^{a_{2}-i-1} P_{1}
$$


can be used for the integral to obtain

$$
\int \frac{\left(k_{\perp}^{2}\right)^{a_{2}} d^{4} k d^{4} l}{P_{1} P_{2} P_{3} P_{4} P_{5} P_{6}}=\int \frac{N_{1}^{a_{2}} d^{4} k d^{4} l}{P_{1} P_{2} P_{3} P_{4} P_{5} P_{6}}-\sum_{i=0}^{a_{2}-1} \int \frac{N_{1}^{i}\left(k_{\perp}^{2}\right)^{a_{2}-i-1}}{P_{2} P_{3} P_{4} P_{5} P_{6}} d^{4} k d^{4} l .
$$

Schematically this reduction reads

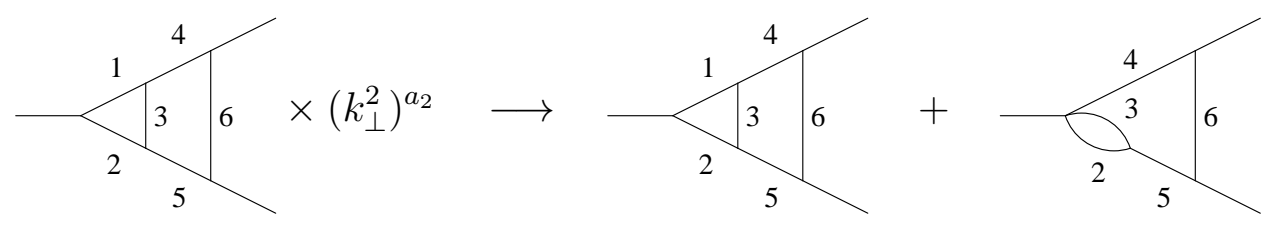

In the following only the diagrammatic reduction will be shown.

\subsection{Replacement of $k_{1}$}

In anticipation of the linearization we replace the numerator factors $k_{1}$ by $\mp\left(\left(k_{0} \mp k_{1}\right)-k_{0}\right)$, using

$$
k_{1}^{a_{1}}=(\mp 1)^{a_{1}} \sum_{i=0}^{a_{1}}\left(-k_{0}\right)^{a_{1}-i}\left(\begin{array}{c}
a_{1} \\
i
\end{array}\right)\left(k_{0} \mp k_{1}\right)^{i} .
$$

While the additional factors $k_{0}^{a_{1}-i}$ will be cancelled together with $k_{0}^{a_{0}}$ in the next step, the subsequent linearization will replace $\left(k_{0} \mp k_{1}\right)$ by $k_{0}^{\prime}$.

\subsection{Cancellation of $k_{0}$}

$P_{1}$ and $P_{2}$ are the only propagator factors that contain only $k$ and not $l$. We can combine these both to obtain

$$
2 E k_{0}=N_{2}+P_{1}-P_{2}, \quad N_{2}=p_{2}^{2}-p_{1}^{2}-m_{2}^{2}+m_{1}^{2}
$$

An iterative formula can be constructed as before, the reduction reads
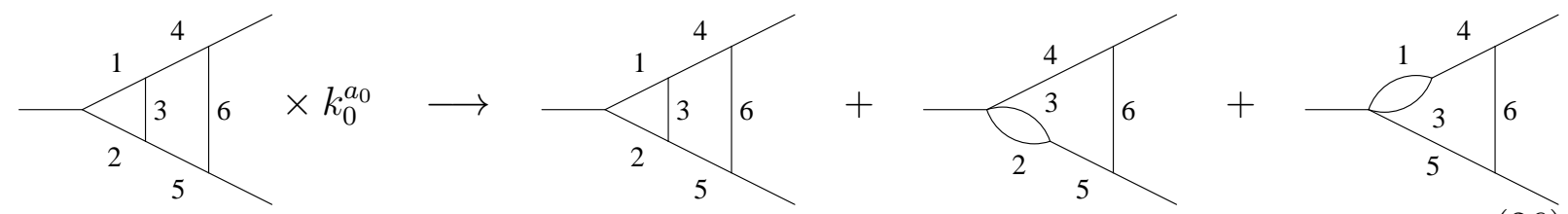

$(26)$ 


\subsection{Cancellation of $l_{\perp}^{2}$}

In using

$$
l_{\perp}^{2}=N_{6}-P_{6}, \quad N_{6}=l_{0}^{2}-l_{1}^{2}-m_{6}^{2}+i \eta
$$

we can cancel the factors $l_{\perp}^{2}$, ending up with the reduction scheme

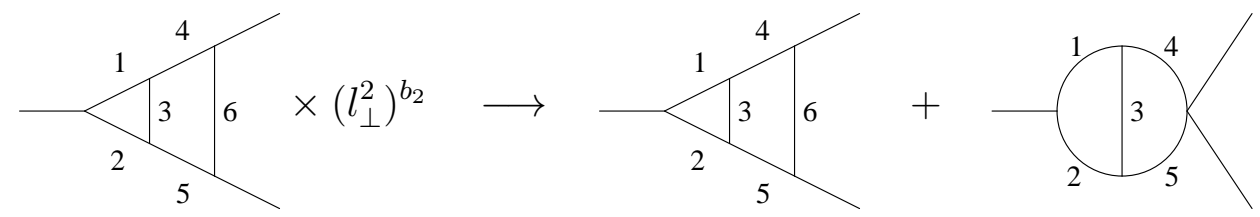

\subsection{Cancellation of $l_{1}$}

Different from the situation for the replacement of $k_{1}$, we have three propagator factors which do not contain $k$. In this case the factors $l_{1}$ can be cancelled completely. We use

$$
2 q_{z} l_{1}=N_{4}+P_{4}-P_{6}, \quad N_{4}=2 l_{0} E_{1}-p_{1}^{2}+m_{4}^{2}-m_{6}^{2}
$$

to reduce powers of $l_{1}$ according to the scheme
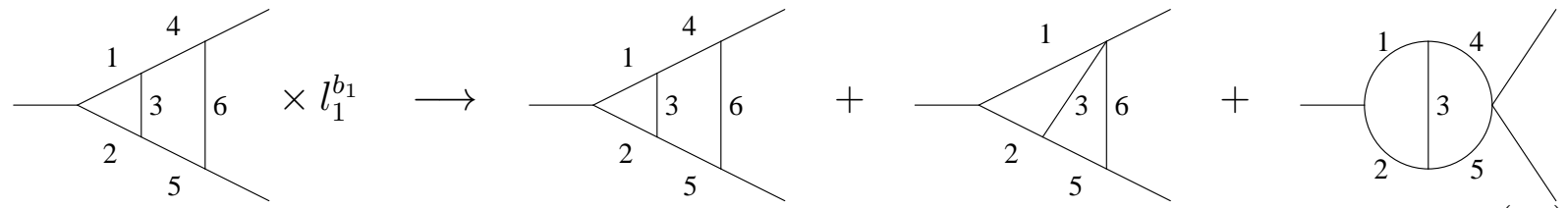

\subsection{Cancellation of $l_{0}$}

Finally, we can cancel the factors $l_{0}$ as well, using

$$
2 E l_{0}=N_{5}+P_{5}-P_{4}, \quad N_{5}=p_{1}^{2}-p_{2}^{2}-m_{4}^{2}+m_{5}^{2} .
$$

The reduction scheme reads
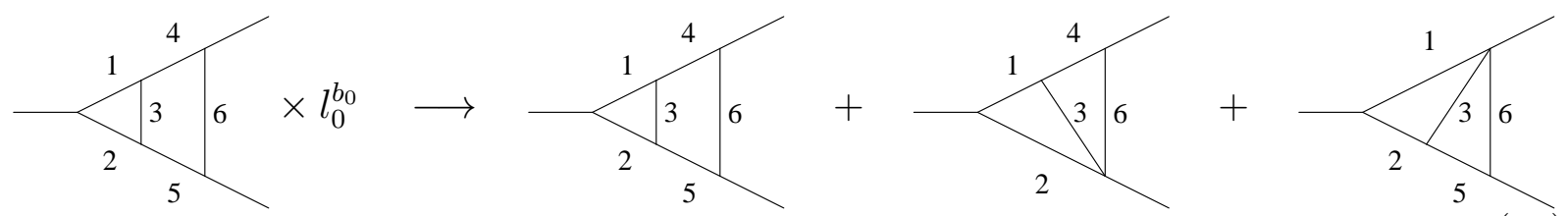


\subsection{Summary of the reduction procedure}

After performing the reduction procedure, we can collect all the steps into a single one. All diagrams which are still of the (original) planar topology after reduction procedure have a numerator factor $\left(k_{0} \mp k_{1}\right)$ in different powers. The maximal power, i.e. the maximal value for $\alpha$, is given by $a_{1}+2 a_{2}+2 c$. The numerator factors of the diagrams with reduced topology are changed but not easier. Nevertheless, because of the different topology, reduction procedures which are known in the literature or which will be explained in the following can be applied. Schematically we write

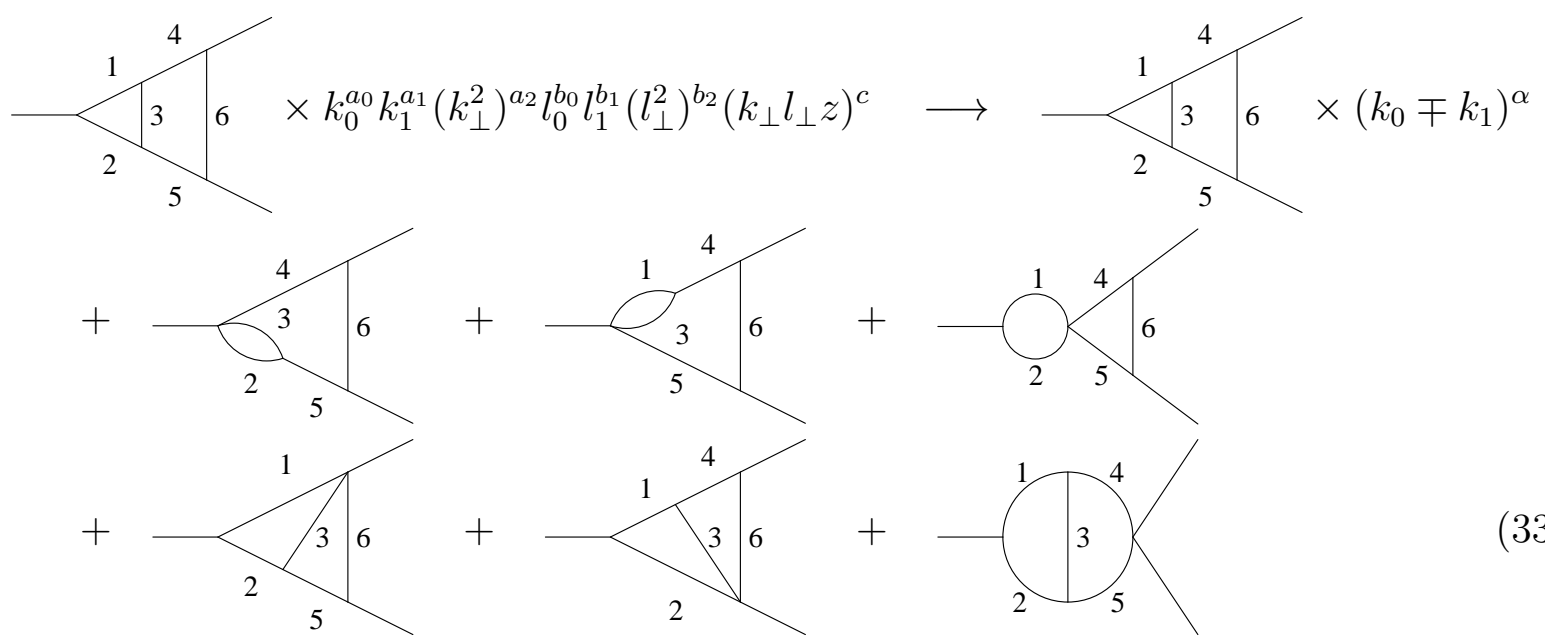

Denoting the topologies by $\mathcal{T}^{0}, \mathcal{T}^{1}, \ldots, \mathcal{T}^{6}$ according to the cancelled propagator factor, we can cite the following references:

- Two-loop three-point functions with two-point subloop, as they are given in our case by the topologies $\mathcal{T}^{1}$ and $\mathcal{T}^{2}$, can be calculated by using dispersion relations [24, 25, 26].

- The factorizing topology $\mathcal{T}^{3}$ is calculated as a product of one-loop topologies. This contributions may be calculated using xloops [50] or similar packages like SANC [56].

- The effective two-loop two-point topology $\mathcal{T}^{6}$ can be calculated using xloops [27].

- What is left are the rotated reduced planar topologies $\mathcal{T}^{4}$ and $\mathcal{T}^{5}$. They can be reduced in a similar manner as the original planar topologies. ${ }^{2}$ In the following section we will deal with these topologies and the necessary modifications of the reduction procedure.

\footnotetext{
${ }^{2}$ Because of historical reasons, these topologies are called rotated reduced planar topologies. In the original reduced planar topology the line crossing the triangle is going horizontally from left to right, connecting the left vertex with the middle of the vertical line on the right.
} 


\subsection{Modifications for the rotated reduced planar topologies}

For the rotated reduced planar topologies we assume the same numerator factor as for the non-reduced one, $k_{0}^{a_{0}} k_{1}^{a_{1}}\left(k_{\perp}^{2}\right)^{a_{2}} l_{0}^{b_{0}} l_{1}^{b_{1}}\left(l_{\perp}^{2}\right)^{b_{2}}\left(k_{\perp} l_{\perp} z\right)^{c}$. The procedure is quite the same as in the case discussed before. But because one of the denominator factors $P_{4}$ and $P_{5}$ is absent, it is not possible to cancel the factors of $l_{1}$ as in the previous case. Instead, we anticipate the linearization as in the case of $k_{1}$ (and with the same sign) in writing

$$
l_{1}=\mp\left(\left(l_{0} \mp l_{1}\right)-l_{0}\right) \quad \Rightarrow \quad l_{1}^{b_{1}}=(\mp 1)^{b_{1}} \sum_{j=0}^{b_{1}}\left(-l_{0}\right)^{b_{1}-j}\left(\begin{array}{c}
b_{1} \\
j
\end{array}\right)\left(l_{0} \mp l_{1}\right)^{j} \text {. }
$$

Again, while the additional factors $l_{0}^{b_{1}-j}$ will be cancelled together with $l_{0}^{b_{0}}$ in the last step, the subsequent linearization will replace $\left(l_{0} \mp l_{1}\right)$ by $l_{0}^{\prime}$. However, this last step makes the difference between the two topologies.

For the topology $\mathcal{T}^{4}$ the denominator factor $P_{4}$ is absent. However, we can obtain

$$
2\left(E_{2} \pm q_{z}\right) l_{0}=N_{5}^{\prime}-P_{6}+P_{5}, \quad N_{5}^{\prime}= \pm 2\left(l_{0} \mp l_{1}\right) q_{z}-p_{2}^{2}-m_{6}^{2}+m_{5}^{2} .
$$

At this point the different signs for the linearization enter the game. While we have designed the term $\left(l_{0} \mp l_{1}\right)$ in the way that it can be combined with the factors from the cancellation of $l_{1}$, in order to obtain $l_{0}$ we have to divide by $\left(E_{2} \pm q_{z}\right)$. However, if the second produced particle is massless, $p_{2}^{2}=0$, this factor will become zero. In order to include also the massless case, we will prefer the linearization $l_{1}=l_{1}^{\prime}+l_{0}$ for this topology, i.e. the upper sign. The whole reduction procedure for the case of the topology $\mathcal{T}^{4}$ reads

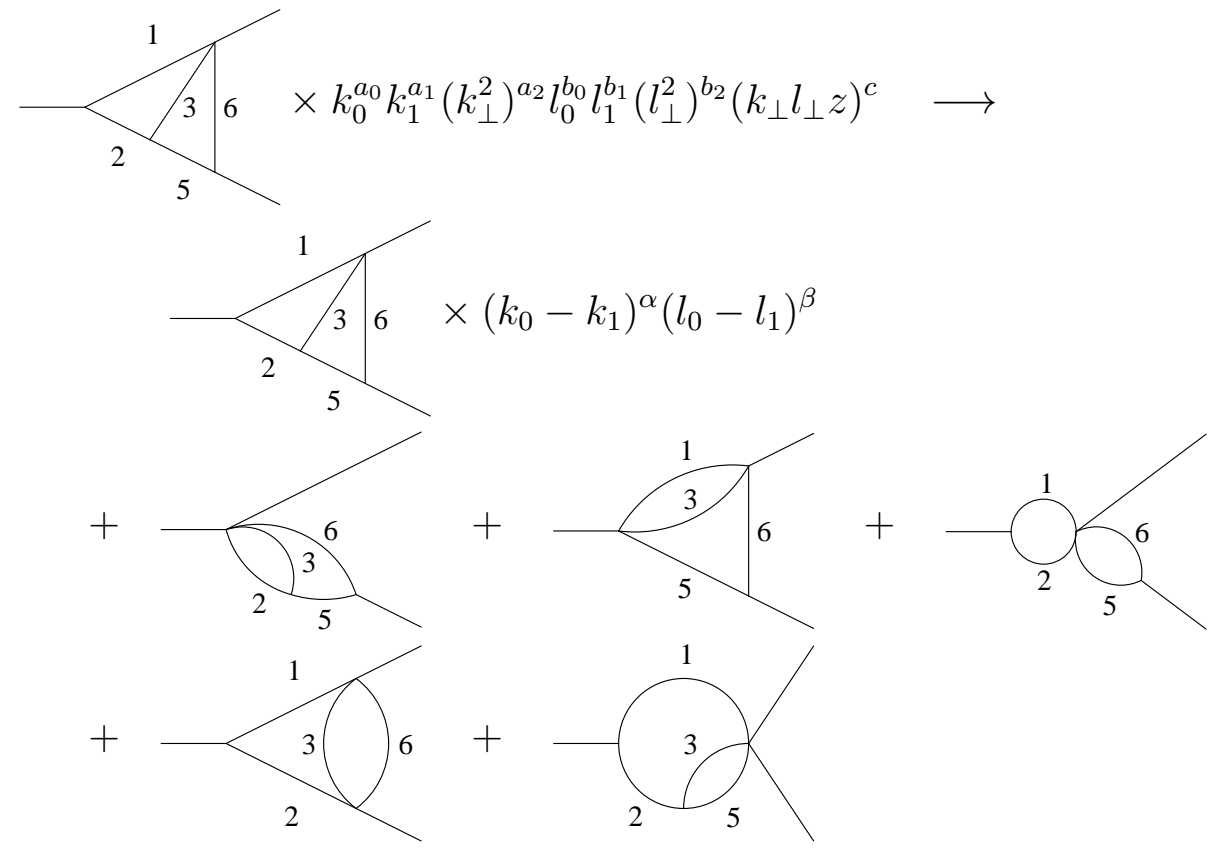


All the diagrams on the right hand side except for the first one with maximal powers $a_{1}+2 a_{2}+2 c$ for $\alpha$ and $b_{0}+b_{1}+2 b_{2}+2 c$ for $\beta$ are again members of the different simpler topology classes which were mentioned in the previous paragraph.

Looking at the topology $\mathcal{T}^{5}$, the propagator factor $P_{5}$ is absent. In this case we can obtain a reduction formula from combining $P_{4}$ and $P_{6}$ to obtain

$$
2\left(E_{1} \mp q_{z}\right) l_{0}=N_{4}^{\prime}-P_{4}+P_{6}, \quad N_{4}^{\prime}=\mp 2\left(l_{0} \mp l_{1}\right) q_{z}+p_{2}^{2}-m_{4}^{2}+m_{6}^{2} .
$$

In this case the linearization $l_{1}=l_{1}^{\prime}-l_{0}$ (lower sign) is more appropriate. The result of the reduction reads

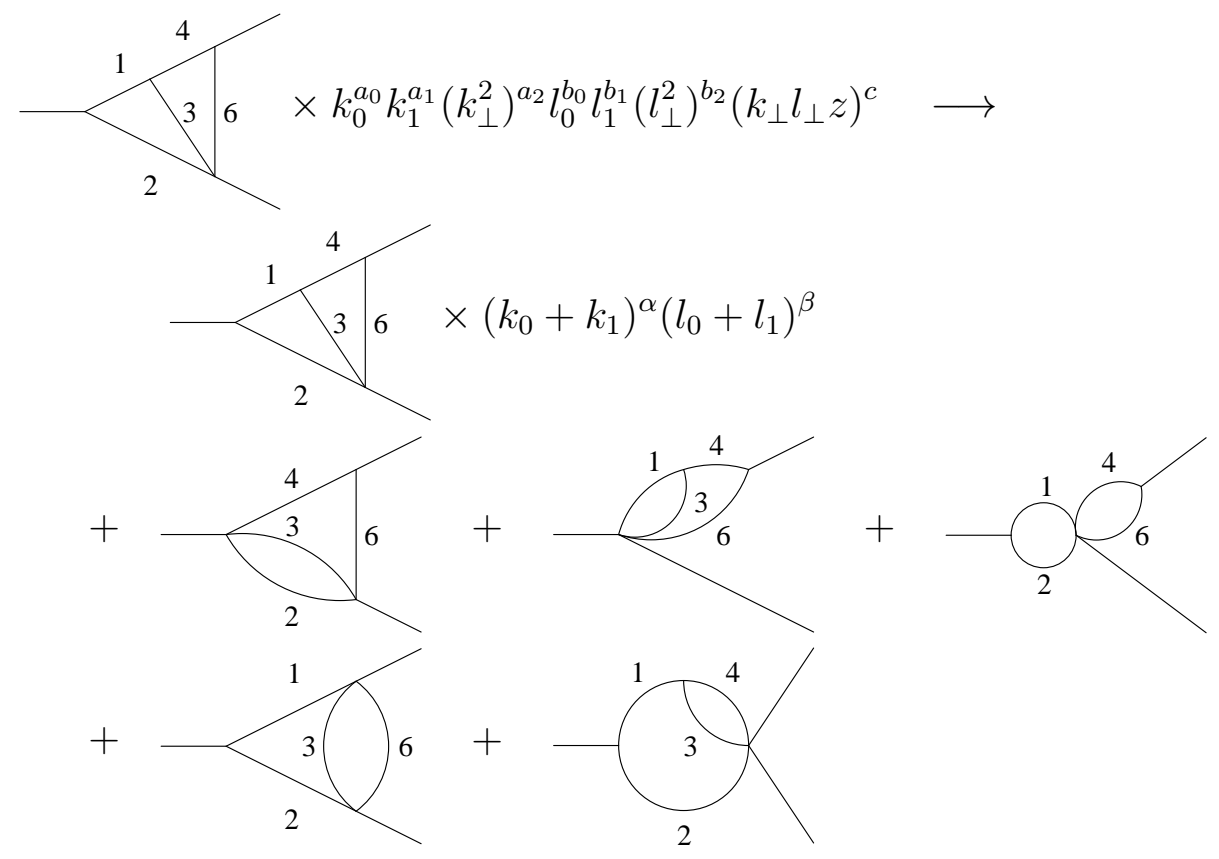

\section{Integration of the master integrals}

After having reduced the numerator of the integrand to factors $\left(k_{0} \mp k_{1}\right)^{\alpha}$ in case of the planar two-loop topology and $\left(k_{0} \mp k_{1}\right)^{\alpha}\left(l_{0} \mp l_{1}\right)^{\beta}$ in case of the rotated reduced planar twoloop topologies, we can start to integrate this set of master integrals $\mathcal{T}_{\alpha}^{0}, \mathcal{T}_{\alpha, \beta}^{x}$. Before we do so, we have to consider the occurrence of ultraviolet (UV) divergences and their treatment in terms of appropriate subtractions. As for the treatment of infrared (IR) divergences we refer to Ref. [41] for the scalar case. In the more general case, methods taken from Ref. [57] can be applied. However, in this paper we consider only IR-finite examples. 


\subsection{UV-divergences and subtraction procedure}

The UV-divergences can be subdivided into three classes: divergences with respect to the two loop momenta and global divergences. It is quite obvious that if we have $d_{k}$ propagator factors depending only on $k, d_{l}$ propagator factors depending only $l, d_{k l}$ propagator factors depending both on $k$ and $l$, and a power $k^{n_{k}} l^{n_{l}}$ in the numerator, the corresponding degrees of divergence in $D$ space-time dimensions are given by

$$
\begin{aligned}
\omega_{k} & =2\left(d_{k}+d_{k l}\right)-n_{k}-D \\
\omega_{l} & =2\left(d_{l}+d_{k l}\right)-n_{l}-D \\
\omega & =2\left(d_{k}+d_{l}+d_{k l}\right)-\left(n_{k}+n_{l}\right)-2 D .
\end{aligned}
$$

The two-loop integral is divergent in $D$ dimensions, if at least one of the degrees $\omega_{k}, \omega_{l}$, or $\omega$ is zero or negative. If the degree of divergence is zero, this divergence is called logarithmic divergence.

In order to calculate the integral, we first have to regularize it. We use dimensional regularization and write $D=4-2 \varepsilon$. After that, integrals can be split off into a divergent and a convergent part. While the convergent part can be calculated for $\varepsilon=0$, i.e. for $D=4$ space-time dimensions, the integrand of the divergent part is simpler but has the same UVbehaviour. In an appropriate subtraction procedure, therefore, we subtract and add an integrand which is simple enough to be integrated analytically (at least the singular part) but has the same singularities as the integrand itself in order to cancel the singularities. This subtraction procedure is found and will be formulated multiplicatively [36, 42]. The subtracted integrand contains the subtraction factors

$$
\mathcal{K}^{\left(j_{k}\right)}=\prod_{r=1}^{j_{k}}\left(1-\frac{D_{k}}{D_{k, r}}\right), \quad \mathcal{L}^{\left(j_{l}\right)}=\prod_{r=1}^{j_{l}}\left(1-\frac{D_{l}}{D_{l, r}}\right)
$$

with

$$
\begin{array}{cc}
D_{k}=\prod_{i=1}^{d_{k}}\left(\left(k+p_{k, i}\right)^{2}-m_{k, i}^{2}+i \eta\right), & D_{k, r}=\prod_{i=1}^{d_{k}}\left(\left(k+\kappa_{r} p_{k, i}\right)^{2}-m_{k, i, r}^{2}+i \eta\right) \\
D_{l}=\prod_{i=1}^{d_{l}}\left(\left(l+p_{l, i}\right)^{2}-m_{l, i}^{2}+i \eta\right), & D_{l, r}=\prod_{i=1}^{d_{l}}\left(\left(l+\lambda_{r} p_{l, i}\right)^{2}-m_{l, i, r}^{2}+i \eta\right)
\end{array}
$$

where $p_{k, i}, p_{l, i} \in\left\{ \pm p_{1}, \pm p_{2}\right\}, m_{k, i} \in\left\{m_{1}, m_{2}\right\}, m_{l, i} \in\left\{m_{4}, m_{5}\right\}$, and $m_{k, i, r}, m_{l, i, r}$ are subtraction masses which have to be introduced artificially. ${ }^{3}$ The degrees of divergence for this

\footnotetext{
${ }^{3}$ In the following we will simplify the indices according to the special cases.
} 
subtracted integrand increase by $j_{k}$ and $j_{l}$, resp. and thus may lead to integrals without singularities for arbitrary values for $\kappa_{r}, \lambda_{r}, m_{k, i, r}$, and $m_{l, i, r}$. Finally, the limit $\kappa_{r}, \lambda_{r} \rightarrow 0$ can be performed, leaving the subtracted integral convergent and enabling the semi-analytical calculation. Since the subtraction terms always contain a two-point subloop, the singular part can be calculated analytically. For the non-singular part one can use $\varepsilon=0$ [24, 25, 26]. In the end the sum of subtracted integral and subtraction terms have to be independent of the subtraction masses. In the following we will deal with the subtracted integrals only.

\subsection{Subtraction for the planar topology}

The reduction procedure explained in the previous section leads to the integrals

$$
\mathcal{T}_{\alpha}^{0}=\int \frac{\left(k_{0} \mp k_{1}\right)^{\alpha}}{P_{1} P_{2} P_{3} P_{4} P_{5} P_{6}} d^{D} k d^{D} l .
$$

The degrees of divergence can be calculated in four space-time dimensions,

$$
\omega_{k}=2-\alpha, \quad \omega_{l}=4, \quad \omega=4-\alpha .
$$

There are no subdivergences in $l$, i.e. divergences caused by the integration over the loop momentum $l$. However, for $\alpha \geq 2$ we have to subtract subdivergences in $k$. We choose $j_{k}=\alpha-1$ and multiply the integrand in Eq. (42) by

$$
\mathcal{K}^{\left(j_{k}\right)}=\prod_{i=1}^{j_{k}}\left(1-\frac{P_{1} P_{2}}{P_{1, i} P_{2, i}}\right)
$$

where $P_{1}=\left(k+p_{1}\right)^{2}-m_{1}^{2}+i \eta$ and $P_{2}=\left(k-p_{2}\right)^{2}-m_{2}^{2}+i \eta$ are given in Eq. (13). The modified propagator factors read

$$
P_{1, i}=\left(k+\kappa_{i} p_{1}\right)^{2}-m_{1, i}^{2}+i \eta, \quad P_{2, i}=\left(k-\kappa_{i} p_{2}\right)^{2}-m_{2, i}^{2}+i \eta .
$$

Note that each of the subtraction factors in Eq. (44) improves the degree of divergence at least by 1 because by power counting the denominator is given by $k^{4}$ and terms of lower order in $k$ while the numerator starts with $k^{3}$. The masses $m_{1, i}, m_{2, i}$ of the subtraction can be chosen arbitrarily. However, in order to avoid the introduction of spurious IRsingularities and squared propagator factors in the denominator, the subtraction masses are usually chosen to be non-zero and different from the physical ones and from each other [42]. Furthermore, in order not to introduce new thresholds, they should be larger than the masses of the decaying particles. On the other hand, they should not be too large because of the possible occurence of numerical instabilities. 


\subsection{Integration for the planar topology}

After the linearization $k_{0}=k_{0}^{\prime}+k_{1}$, in the $\mathrm{P} / \mathrm{O}$-space representation the propagator factors are given by

$$
\begin{aligned}
P_{1} & =2\left(k_{0}^{\prime}+E_{1}-q_{z}\right) k_{1}+\left(k_{0}^{\prime}+E_{1}\right)^{2}-q_{z}^{2}-s-m_{1}^{2}+i \eta \\
P_{2} & =2\left(k_{0}^{\prime}-E_{2}-q_{z}\right) k_{1}+\left(k_{0}^{\prime}-E_{2}\right)^{2}-q_{z}^{2}-s-m_{2}^{2}+i \eta \\
P_{1, i} & =2\left(k_{0}^{\prime}-\kappa_{i}\left(E_{1}-q_{z}\right)\right) k_{1}+\left(k_{0}^{\prime}+\kappa_{i} E_{1}\right)^{2}-\kappa_{i}^{2} q_{z}^{2}-s-m_{1, i}^{2}+i \eta \\
P_{2, i} & =2\left(k_{0}^{\prime}-\kappa_{i}\left(E_{2}+q_{z}\right)\right) k_{1}+\left(k_{0}^{\prime}-\kappa_{i} E_{2}\right)^{2}-\kappa_{i}^{2} q_{z}^{2}-s-m_{2, i}^{2}+i \eta .
\end{aligned}
$$

In case of the (non-reduced) planar diagram where both linearizations are possible, we select the linearizations $k_{0}=k_{0}^{\prime}+k_{1}$ and $l_{0}=l_{0}^{\prime}+l_{1}$ for convenience. The propagator factors which are linear expressions in $k_{1}$ can be written in closed form expressions,

$$
\begin{array}{ll}
P_{1}=\theta_{1} k_{1}+\xi_{1}-s+i \eta, & P_{1, i}=\theta_{1, i} k_{1}+\xi_{1, i}-s+i \eta \\
P_{2}=\theta_{2} k_{1}+\xi_{2}-s+i \eta, & P_{2, i}=\theta_{2, i} k_{1}+\xi_{2, i}-s+i \eta
\end{array}
$$

Because the parameters $\kappa_{i}$ can take arbitrary values, the denominator of $\mathcal{K}^{\left(j_{k}\right)}$ in Eq. (40) together with the factors $P_{1}$ and $P_{2}$ from the integrand consists of different linear factors.

Integrating over $k_{1}$, the integration path $[-\infty,+\infty]$ can be closed in the upper or lower complex half plane, and Cauchy's theorem can be applied. The decision for one of these paths depends on the sign of the linearization and on the sign of $\left(k_{0}^{\prime}+l_{0}^{\prime}\right)$ (cf. Eq. (11) and its discussion). The poles are given by the (different) zeros of the propagator factors present in the calculation, the residues are given by

$$
\begin{aligned}
& \operatorname{Res}\left[\frac{1}{P_{1} P_{2}} \prod_{i=1}^{j_{k}}\left(1-\frac{P_{1} P_{2}}{P_{1, i} P_{2, i}}\right) ; P_{1}=0\right]=\left.\frac{1}{P_{2}}\right|_{P_{1}=0}, \\
& \operatorname{Res}\left[\frac{1}{P_{1} P_{2}} \prod_{i=1}^{j_{k}}\left(1-\frac{P_{1} P_{2}}{P_{1, i} P_{2, i}}\right) ; P_{2}=0\right]=\left.\frac{1}{P_{1}}\right|_{P_{2}=0}, \\
& \operatorname{Res}\left[\frac{1}{P_{1} P_{2}} \prod_{i=1}^{j_{k}}\left(1-\frac{P_{1} P_{2}}{P_{1, i} P_{2, i}}\right) ; P_{1, j}=0\right]=-\left.\frac{1}{P_{2, j}} \prod_{\substack{i=1 \\
i \neq j}}^{j_{k}}\left(1-\frac{P_{1} P_{2}}{P_{1, i} P_{2, i}}\right)\right|_{P_{1, j}=0}, \\
& \operatorname{Res}\left[\frac{1}{P_{1} P_{2}} \prod_{i=1}^{j_{k}}\left(1-\frac{P_{1} P_{2}}{P_{1, i} P_{2, i}}\right) ; P_{2, j}=0\right]=-\left.\frac{1}{P_{1, j}} \prod_{\substack{i=1 \\
i \neq j}}^{j_{k}}\left(1-\frac{P_{1} P_{2}}{P_{1, i} P_{2, i}}\right)\right|_{P_{2, j}=0} .
\end{aligned}
$$

The condition $P_{1}=0$ results in $k_{1}=\left(s-\xi_{1}-i \eta\right) / \theta_{1}=: k_{1(1)}$. One obtains

$$
\left.\frac{1}{P_{2}}\right|_{P_{1}=0}=\frac{\theta_{1}}{\left(\theta_{2}-\theta_{1}\right)\left(s-s_{2,1}-i \eta\right)}=\frac{\mathcal{N}_{1}^{k}}{s-s_{2,1}-i \eta}, \quad s_{2,1}:=\frac{\theta_{2} \xi_{1}-\theta_{1} \xi_{2}}{\theta_{2}-\theta_{1}}
$$


In the same way $P_{2}=0$ results in $k_{1}=\left(s-\xi_{2}-i \eta\right) / \theta_{2}=: k_{1(2)}$. One obtains

$$
\left.\frac{1}{P_{1}}\right|_{P_{2}=0}=\frac{\theta_{2}}{\left(\theta_{1}-\theta_{2}\right)\left(s-s_{1,2}-i \eta\right)}=\frac{\mathcal{N}_{2}^{k}}{s-s_{1,2}-i \eta}, \quad s_{1,2}:=\frac{\theta_{1} \xi_{2}-\theta_{2} \xi_{1}}{\theta_{1}-\theta_{2}} .
$$

Note that $s_{1,2}=s_{2,1}$. For the other two types of residues, however, a simplification appears after inserting the poles. Because of the fact that for the semi-analytical calculation of the convergent part of the integral we use the limit $\kappa_{i} \rightarrow 0$ for all $i$, we obtain

$$
\theta_{1, i}, \theta_{2, i} \rightarrow 2 k_{0}=: \theta_{0} \quad \text { for } i=1,2, \ldots, j_{k} .
$$

In this limit, and on the pole $P_{1, j}=0$ given by $k_{1}=\left(s-\xi_{1, j}-i \eta\right) / \theta_{0}=: k_{1(1, j)}$, the different propagator factors read

$$
\begin{aligned}
\left.P_{1, i}\right|_{P_{1, j}=0} & =\xi_{1, i}-\xi_{1, j}, \\
\left.P_{2, i}\right|_{P_{1, j}=0} & =\xi_{2, i}-\xi_{1, j}, \\
\left.P_{1}\right|_{P_{1, j}=0} & =\frac{1}{\theta_{0}}\left(\left(\theta_{1}-\theta_{0}\right)(s-i \eta)+\left(\xi_{1} \theta_{0}-\theta_{1} \xi_{1, j}\right)\right), \\
\left.P_{2}\right|_{P_{1, j}=0} & =\frac{1}{\theta_{0}}\left(\left(\theta_{2}-\theta_{0}\right)(s-i \eta)+\left(\xi_{2} \theta_{0}-\theta_{2} \xi_{1, j}\right)\right) .
\end{aligned}
$$

Obviously, the dependence on $s$ appears only in the numerator. We can write

$$
-\left.\frac{1}{P_{2, j}} \prod_{\substack{i=1 \\ i \neq j}}^{j_{k}}\left(1-\frac{P_{1} P_{2}}{P_{1, i} P_{2, i}}\right)\right|_{P_{1, j}=0}=\sum_{a=0}^{2 j_{k}-2} \mathcal{N}_{1, j, a}^{k} s^{a}
$$

and similarly

$$
-\left.\frac{1}{P_{1, j}} \prod_{\substack{i=1 \\ i \neq j}}^{j_{k}}\left(1-\frac{P_{1} P_{2}}{P_{1, i} P_{2, i}}\right)\right|_{P_{2, j}=0}=\sum_{a=0}^{2 j_{k}-2} \mathcal{N}_{2, j, a}^{k} s^{a} .
$$

The different poles are given by

$$
\begin{aligned}
& k_{1(1)}=\frac{s-\xi_{1}-i \eta}{\theta_{1}} \quad k_{1(2)}=\frac{s-\xi_{2}-i \eta}{\theta_{2}} \\
& k_{1(1, i)}=\frac{s-\xi_{1, i}-i \eta}{\theta_{0}} \quad k_{2(2, i)}=\frac{s-\xi_{2, i}-i \eta}{\theta_{0}} \quad \text { for } i=1,2, \ldots, j_{k} .
\end{aligned}
$$

For $\theta_{i}<0(i=0,1,2)$ the corresponding pole can be found in the upper complex half plane. For the linearization $k_{0}=k_{0}^{\prime}+k_{1}$ and $k_{0}^{\prime}+l_{0}^{\prime}>0$, the path has to be closed in the upper half plane. In this case we obtain a non-vanishing residue. For $k_{0}^{\prime}+l_{0}^{\prime}<0$, however, the residue occurs only in case of $\theta_{i}>0$ and has the opposite sign. Using

$$
\mathcal{B}_{n}^{k}=2 \pi i\left[\theta\left(k_{0}^{\prime}+l_{0}^{\prime}\right) \theta\left(-\theta_{n}\right)-\theta\left(-\left(k_{0}^{\prime}+l_{0}^{\prime}\right)\right) \theta\left(\theta_{n}\right)\right], \quad n=0,1,2
$$


where $\theta(x)$ is the step function, we can integrate over $k_{1}$ to obtain

$$
\begin{aligned}
& \int_{-\infty}^{+\infty} \frac{d k_{1}}{P_{1} P_{2}}\left(\prod_{i=1}^{j_{k}}\left(1-\frac{P_{1} P_{2}}{P_{1, i} P_{2, i}}\right)\right) f\left(k_{1}, l_{1}\right) \\
& =\sum_{n=1}^{2}\left(\frac{\mathcal{B}_{n}^{k} \mathcal{N}_{n}^{k}}{s-s_{1,2}-i \eta} f\left(k_{1(n)}, l_{1}\right)+\sum_{i=1}^{j_{k}} \sum_{a=0}^{2 j_{k}-2} \mathcal{B}_{0}^{k} \mathcal{N}_{n, i, a}^{k} s^{a} f\left(k_{1(n, i)}, l_{1}\right)\right)
\end{aligned}
$$

where $f\left(k_{1}, l_{1}\right)$ is given e.g. by the inverse of $\sqrt[*]{\left(A\left(k_{1}, l_{1}\right)+i \eta\right)^{2}-4 s t}$ (cf. Eq. (10) $)$.

For the integration over $l_{1}$ we have to consider the propagator factors $P_{4}, P_{5}$, and $P_{6}$. Because the degree of divergence $\omega_{l}$ is positive, no subtractions are needed. After the linearization $l_{0}=l_{0}^{\prime}+l_{1}$ we can write

$$
\begin{aligned}
P_{4} & =2\left(l_{0}^{\prime}-E_{1}+q_{z}\right) l_{1}+\left(l_{0}^{\prime}-E_{1}\right)^{2}-q_{z}^{2}-t-m_{4}^{2}+i \eta \\
P_{5} & =2\left(l_{0}^{\prime}+E_{2}+q_{z}\right) l_{1}+\left(l_{0}^{\prime}+E_{2}\right)^{2}-q_{z}^{2}-t-m_{5}^{2}+i \eta \\
P_{6} & =2 l_{0}^{\prime} l_{1}+l_{0}^{\prime 2}-t-m_{6}^{2}+i \eta
\end{aligned}
$$

which again can be cast into the closed form expression

$$
P_{i}=\phi_{i} l_{1}+\psi_{i}-t+i \eta, \quad i=4,5,6
$$

The procedure which was explained for the integration over $k_{1}$ works accordingly. The poles of

$$
\frac{1}{P_{4} P_{5} P_{6}}=\left(\prod_{j=4}^{6}\left(\phi_{j} l_{1}+\psi_{j}-t+i \eta\right)\right)^{-1}
$$

are found at

$$
l_{1(m)}=\frac{t-\psi_{m}-i \eta}{\phi_{m}} \quad \text { for } m=4,5,6 .
$$

From Eq. (11) we read off that the cut is located in the same complex half plane as in case of the integration over $k_{1}$. Therefore, the occurrence and the sign of the residue is determined by

$$
\mathcal{B}_{m}^{l}=2 \pi i\left[\theta\left(k_{0}^{\prime}+l_{0}^{\prime}\right) \theta\left(-\phi_{m}\right)-\theta\left(-\left(k_{0}^{\prime}+l_{0}^{\prime}\right)\right) \theta\left(\phi_{m}\right)\right], \quad m=4,5,6 .
$$

Using Cauchy's theorem, we obtain

$$
\int_{-\infty}^{+\infty} \frac{d l_{1}}{P_{4} P_{5} P_{6}} f\left(k_{1}, l_{1}\right)
$$




$$
\begin{aligned}
& =\sum_{m=4}^{6}\left(\prod_{\substack{j=4 \\
j \neq m}}^{6}\left(\phi_{j} l_{1(m)}+\psi_{j}-t+i \eta\right)\right)^{-1} \mathcal{B}_{m}^{l} f\left(k_{1}, l_{1(m)}\right) \\
& =\sum_{m=4}^{6}\left(\prod_{\substack{j=4 \\
j \neq m}}^{6}\left(t\left(\phi_{j}-\phi_{m}\right)+\left(\psi_{j} \phi_{m}-\psi_{m} \phi_{j}\right)-i \eta\left(\phi_{j}-\phi_{m}\right)\right)\right)^{-1} \phi_{m}^{2} \mathcal{B}_{m}^{l} f\left(k_{1}, l_{1(m)}\right) .
\end{aligned}
$$

Of course, the same is valid if $k_{1}$ is replaced by $k_{1(n)}$ or $k_{1(n, i)}$. Usually, $\phi_{j}$ and $\phi_{m}$ are different for $j \neq m$. In this case we can write

$$
t\left(\phi_{j}-\phi_{m}\right)-\left(\phi_{j} \psi_{m}-\phi_{m} \psi_{j}\right)-i \eta\left(\phi_{j}-\phi_{m}\right)=\left(\phi_{j}-\phi_{m}\right)\left(t-t_{j, m}-i \eta\right)
$$

with

$$
t_{j, m}=\frac{\phi_{j} \psi_{m}-\phi_{m} \psi_{j}}{\phi_{j}-\phi_{m}}
$$

We perform a partial fraction decomposition to obtain

$$
\int_{-\infty}^{+\infty} \frac{d l_{1}}{P_{4} P_{5} P_{6}} f\left(k_{1}, l_{1}\right)=\sum_{m=4}^{5} \sum_{j=m+1}^{6} \frac{\mathcal{B}_{m}^{l} \mathcal{N}_{m, j}^{l}}{t-t_{m, j}-i \eta} f\left(k_{1}, l_{1(m)}\right)
$$

where $\mathcal{N}_{m, j}^{l}$ are the corresponding coefficients including $\phi_{m}^{2}$. A special situation occurs if $p_{1}^{2}=0$. Because of $q_{z}=E_{1}$, in this case we have $\phi_{4}=\phi_{6}$ and the corresponding denominator factor contributes in the form $\left(\psi_{4}-\psi_{6}\right) \phi_{6}$ only. However, if $p_{2}^{2}=0$, no special case has to be taken. ${ }^{4}$

The integrations over $k_{1}$ and $l_{1}$ and the integration over $z$ can be combined in the closed form expression for the convergent part $\mathcal{V}_{\alpha}^{0}$ of the master integral $\mathcal{T}_{\alpha}^{0}$ in case of $\kappa_{i} \rightarrow 0$,

$$
\begin{aligned}
\mathcal{V}_{\alpha}^{0}= & \int \frac{\left(k_{0}-k_{1}\right)^{\alpha}}{P_{1} P_{2} P_{3} P_{4} P_{5} P_{6}} \mathcal{K}^{\left(j_{k}\right)} d^{4} k d^{4} l=\pi^{2} \sum_{n=1}^{2} \sum_{m=4}^{5} \sum_{j=m+1}^{6} \int_{0}^{\infty} k_{0}^{\prime \alpha} d k_{0}^{\prime} d l_{0}^{\prime} \int_{0}^{\infty} \frac{\mathcal{N}_{m, j}^{l} d t}{t-t_{m, j}-i \eta} \\
& \times \int_{0}^{\infty}\left(\frac{\mathcal{B}_{n, m}^{k l} \mathcal{N}_{n}^{k} d s}{\left(s-s_{1,2}-i \eta\right) \sqrt[\star]{\left(A_{n, m}+i \eta\right)^{2}-4 s t}}+\sum_{i=1}^{j_{k}} \sum_{a=0}^{2 j_{k}-2} \frac{\mathcal{B}_{0, m}^{k l} \mathcal{N}_{n, i, a}^{k} s^{a} d s}{\sqrt[\star]{\left(A_{n, i, m}+i \eta\right)^{2}-4 s t}}\right)
\end{aligned}
$$

where

$$
\mathcal{B}_{n, m}^{k l}=\mathcal{B}_{n}^{k} \mathcal{B}_{m}^{l}=-4 \pi^{2}\left[\theta\left(k_{0}^{\prime}+l_{0}^{\prime}\right) \theta\left(-\theta_{n}\right) \theta\left(-\phi_{m}\right)+\theta\left(-\left(k_{0}^{\prime}+l_{0}^{\prime}\right)\right) \theta\left(\theta_{n}\right) \theta\left(\phi_{m}\right)\right]
$$

and $A_{n, m}, A_{n, i, m}$ are the coefficients $A$ in Eq. (9) with $k_{1}$ replaced by $k_{1(n)}, k_{1(n, i)}$ and $l_{1}$ replaced by $l_{1(m)}$, i.e. $A_{n, m}=A\left(k_{1(n)}, l_{1(m)}\right)$ and $A_{n, i, m}=A\left(k_{1(n, i)}, l_{1(m)}\right)$.

\footnotetext{
${ }^{4}$ For the other linearization $l_{0}=l_{0}^{\prime}-l_{1}$ the situation is the opposite.
} 


\subsection{Subtraction for the rotated reduced planar topologies}

For the rotated reduced planar topologies we are left with the integrals

$$
\mathcal{T}_{\alpha \beta}^{9-x}=\int \frac{\left(k_{0} \mp k_{1}\right)^{\alpha}\left(l_{0} \mp l_{1}\right)^{\beta}}{P_{1} P_{2} P_{3} P_{x} P_{6}} d^{D} k d^{D} l
$$

where in case of the topology $\mathcal{T}^{4}(x=5)$ the upper sign, in case of the topology $\mathcal{T}^{5}(x=4)$ the lower sign is valid. The degrees of divergence can be calculated to be

$$
\omega_{k}=2-\alpha, \quad \omega_{l}=2-\beta, \quad \omega=2-\alpha-\beta .
$$

For $\beta \geq 2$ the integration over $l$ becomes divergent and a subtraction has to be performed in addition to the subtraction in $k$. The subtraction factor is given by

$$
\mathcal{L}^{\left(j_{l}\right)}=\prod_{j=1}^{j_{l}}\left(1-\frac{P_{x}}{P_{x, j}}\right), \quad x=4,5
$$

where in addition to $P_{4}=\left(l-p_{1}\right)^{2}-m_{4}^{2}+i \eta$ and $P_{5}=\left(l+p_{2}\right)^{2}-m_{5}^{2}+i \eta$ we have

$$
P_{4, j}=\left(l-\lambda_{j} p_{1}\right)^{2}-m_{4, j}^{2}+i \eta, \quad P_{5, j}=\left(l+\lambda_{j} p_{2}\right)^{2}-m_{5, j}^{2}+i \eta
$$

The same limitations as they were mentioned at the end of Sec. 4.2 for the subtraction masses $m_{1, i}$ and $m_{2, i}$ apply for the masses $m_{4, j}$ and $m_{5, j}$ as well.

\subsection{Integration for the rotated reduced planar topologies}

The integration over $k_{1}$ can be done in the same way as for the original (non-reduced) planar

topology. However, special care has to be taken concerning the different linearization in case of the topology $\mathcal{T}^{5}$. For the topology $\mathcal{T}^{5}$ we have $\theta_{0}=-2 k_{0}^{\prime}$ and the step function term $\mathcal{B}_{n}^{k}$ has to be replaced by

$$
\mathcal{B}_{n}^{k}=2 \pi i\left[\theta\left(-\left(k_{0}^{\prime}+l_{0}^{\prime}\right)\right) \theta\left(-\theta_{n}\right)-\theta\left(k_{0}^{\prime}+l_{0}^{\prime}\right) \theta\left(\theta_{n}\right)\right]
$$

For the integration over $l_{1}$ we have to perform a subtraction. After the linearization $l_{0}=l_{0}^{\prime} \pm k_{1}$ the propagator factors in the $\mathrm{P} / \mathrm{O}$-space representation are given by

$$
\begin{aligned}
P_{5} & =2\left(l_{0}^{\prime}+\left(E_{2}+q_{z}\right)\right) l_{1}+\left(l_{0}^{\prime}+E_{2}\right)^{2}-q_{z}^{2}-t-m_{5}^{2}+i \eta \\
P_{5, j} & =2\left(l_{0}^{\prime}+\lambda_{j}\left(E_{2}+q_{z}\right)\right) l_{1}+\left(l_{0}^{\prime}+\lambda_{j} E_{2}\right)^{2}-\lambda_{j}^{2} q_{z}^{2}-t-m_{5}^{2}+i \eta \\
P_{6} & =2 l_{0}^{\prime} l_{1}+l_{0}^{\prime 2}-t-m_{6}^{2}+i \eta \quad \text { for the topology } \mathcal{T}_{4}
\end{aligned}
$$




$$
\begin{aligned}
P_{4} & =-2\left(l_{0}^{\prime}-\left(E_{1}+q_{z}\right)\right) l_{1}+\left(l_{0}^{\prime}-E_{1}\right)^{2}-q_{z}^{2}-t-m_{4}^{2}+i \eta \\
P_{4, j} & =-2\left(l_{0}^{\prime}-\lambda_{j}\left(E_{2}+q_{z}\right)\right) l_{1}+\left(l_{0}^{\prime}-\lambda_{j} E_{2}\right)^{2}-\lambda_{j}^{2} q_{z}^{2}-t-m_{4}^{2}+i \eta \\
P_{6} & =-2 l_{0}^{\prime} l_{1}+l_{0}^{\prime 2}-t-m_{6}^{2}+i \eta \quad \text { for the topology } \mathcal{T}_{5} .
\end{aligned}
$$

These propagator factors can be written as

$$
\begin{aligned}
P_{x} & =\phi_{x} l_{1}+\psi_{x}-t+i \eta, \quad P_{x, j}=\phi_{x, j} l_{1}+\psi_{x, j}-t+i \eta \quad x=4,5 \\
P_{6} & =\phi_{6} l_{1}+\psi_{6}-t+i \eta .
\end{aligned}
$$

In the limit $\lambda_{i} \rightarrow 0$, which will be considered in the following, we see that

$$
\phi_{x, j} \rightarrow \phi_{6}= \pm 2 l_{0}^{\prime}
$$

In case of the linearization $l_{0}=l_{0}^{\prime}+l_{1}$ the integration path $[-\infty,+\infty]$ over $l_{1}$ can be closed in the upper complex half plane for $\left(k_{0}^{\prime}+l_{0}^{\prime}\right)>0$ and in the lower complex half plane for $\left(k_{0}^{\prime}+l_{0}^{\prime}\right)<0$. For the other linearization $k_{0}=k_{0}^{\prime}-k_{1}$ the situation is just the opposite. Because the poles are given by

$$
l_{1(x)}=\frac{t-\psi_{x}-i \eta}{\phi_{x}}, \quad l_{1(x, j)}=\frac{t-\psi_{x, j}-i \eta}{\phi_{6}}, \quad l_{1(6)}=\frac{t-\psi_{6}-i \eta}{\phi_{6}}
$$

the integration ranges in $t$ are constrained by

$$
\mathcal{B}_{m}^{l}= \begin{cases}2 \pi i\left[\theta\left(-\left(k_{0}^{\prime}+l_{0}^{\prime}\right)\right) \theta\left(-\phi_{m}\right)-\theta\left(k_{0}^{\prime}+l_{0}^{\prime}\right) \theta\left(\phi_{m}\right)\right] & \text { for } x=4\left(\text { topology } \mathcal{T}^{5}\right) \\ 2 \pi i\left[\theta\left(k_{0}^{\prime}+l_{0}^{\prime}\right) \theta\left(-\phi_{m}\right)-\theta\left(-\left(k_{0}^{\prime}+l_{0}^{\prime}\right)\right) \theta\left(\phi_{m}\right)\right] & \text { for } x=5\left(\text { topology } \mathcal{T}^{4}\right) .\end{cases}
$$

In order to use Cauchy's theorem, we calculate the residues

$$
\begin{aligned}
& \operatorname{Res}\left[\frac{1}{P_{x} P_{6}} \prod_{i=1}^{j_{l}}\left(1-\frac{P_{x}}{P_{x, i}}\right) ; P_{x}=0\right]=\left.\frac{1}{P_{6}}\right|_{P_{x}=0}, \\
& \operatorname{Res}\left[\frac{1}{P_{x} P_{6}} \prod_{i=1}^{j_{l}}\left(1-\frac{P_{x}}{P_{x, i}}\right) ; P_{x, j}=0\right]=\left.\frac{1}{P_{6}} \prod_{\substack{i=1 \\
i \neq j}}\left(1-\frac{P_{x}}{P_{x, i}}\right)\right|_{P_{x, j}=0}, \\
& \operatorname{Res}\left[\frac{1}{P_{x} P_{6}} \prod_{i=1}^{j_{l}}\left(1-\frac{P_{x}}{P_{x, i}}\right) ; P_{6}=0\right]=\left.\frac{1}{P_{x}} \prod_{i=1}^{j_{l}}\left(1-\frac{P_{x}}{P_{x, i}}\right)\right|_{P_{6}=0} .
\end{aligned}
$$

For the first residue we obtain

$$
\left.\frac{1}{P_{6}}\right|_{P_{x}=0}=\frac{\phi_{x}}{\left(\phi_{6}-\phi_{x}\right)\left(t-t_{6, x}-i \eta\right)}=: \frac{\mathcal{N}_{x}^{l}}{t-t_{6, x}-i \eta}, \quad t_{6, x}=\frac{\phi_{6} \psi_{x}-\phi_{x} \psi_{6}}{\phi_{6}-\phi_{x}} .
$$


For the second one we first calculate

$$
\begin{aligned}
\left.P_{6}\right|_{P_{x, j}=0} & =\psi_{6}-\psi_{x, j}, \\
\left.P_{x}\right|_{P_{x, j}=0} & =\frac{1}{\phi_{6}}\left(\left(\phi_{x}-\phi_{6}\right)(t-i \eta)-\left(\phi_{x} \psi_{x, j}-\phi_{6} \psi_{x}\right)\right), \\
\left.P_{x, i}\right|_{p_{x, j}=0} & =\psi_{x, i}-\psi_{x, j} .
\end{aligned}
$$

Therefore, the denominator of this residuum is independent of $t$ while the numerator is a power series up to the power $t^{j_{l}-1}$. We define the coefficients $\mathcal{N}_{x, j, a}^{l}$ by

$$
\left.\frac{1}{P_{6}} \prod_{\substack{i=1 \\ i \neq j}}\left(1-\frac{P_{x}}{P_{x, i}}\right)\right|_{P_{x, j}=0}=: \sum_{a=0}^{j_{l}-1} \mathcal{N}_{x, j, a}^{l} t^{a}
$$

The last residue appears to be a combination of a pole in $t$ and a power series. However, we can separate these two parts by adding and subtracting an appropriate term. This term is given by

$$
\left.\frac{1}{P_{x}}\right|_{P_{6}=0}=\frac{\phi_{6}}{\left(\phi_{x}-\phi_{6}\right)\left(t-t_{x, 6}-i \eta\right)}=: \frac{\mathcal{N}_{6}^{l}}{t-t_{x, 6}-i \eta}, \quad t_{x, 6}=\frac{\phi_{x} \psi_{6}-\phi_{6} \psi_{x}}{\phi_{x}-\phi_{6}} .
$$

If we subtract this term from the result for the last residue, we obtain

$$
\frac{1}{P_{x}} \prod_{i=1}^{j_{l}}\left(1-\frac{P_{x}}{P_{x, i}}\right)-\frac{1}{P_{x}}=\frac{1}{P_{x}}\left\{1-\sum_{i=1}^{j_{l}} \frac{P_{x}}{P_{x, i}}+O\left(P_{x}^{2}\right)\right\}-\frac{1}{P_{x}}=-\sum_{i=1}^{j_{l}} \frac{1}{P_{x, i}}+O\left(P_{x}\right) .
$$

The difference no longer has a factor $P_{x}$ in the denominator. Instead, we obtain again a power series up to the power $t^{j_{l}-1}$. We define the coefficients $\mathcal{N}_{6, j, a}^{l}$ by

$$
\left.\frac{1}{P_{x}} \prod_{i=1}^{j_{l}}\left(1-\frac{P_{x}}{P_{x, i}}\right)\right|_{P_{6}=0}-\left.\frac{1}{P_{x}}\right|_{P_{6}=0}=: \sum_{a=0}^{j_{l}-1} \mathcal{N}_{6, j, a}^{l} t^{a} .
$$

Having calculated the residues, we can perform the integration over $l_{1}$ to obtain

$$
\begin{aligned}
\int_{-\infty}^{+\infty} & \frac{d l_{1}}{P_{x} P_{6}} \prod_{i=1}^{j_{l}}\left(1-\frac{P_{x}}{P_{x, i}}\right) f\left(k_{1}, l_{1}\right) \\
= & \frac{\mathcal{B}_{x}^{l} \mathcal{N}_{x}^{l}}{t-t_{6, x}-i \eta} f\left(k_{1}, l_{1(x)}\right)+\frac{\mathcal{B}_{6}^{l} \mathcal{N}_{6}^{l}}{t-t_{x, 6}-i \eta} f\left(k_{1}, l_{1(6)}\right) \\
& \quad+\sum_{i=1}^{j_{l}} \sum_{a=0}^{j_{l}-1}\left(\mathcal{B}_{6}^{l} \mathcal{N}_{x, i, a}^{l} t^{a}+\mathcal{B}_{6}^{l} \mathcal{N}_{6, i, a}^{l} t^{a}\right) f\left(k_{1}, l_{1(6, i)}\right) \\
= & \sum_{m=x, 6}\left(\frac{\mathcal{B}_{m}^{l} \mathcal{N}_{m}^{l}}{t-t_{x, 6}-i \eta} f\left(k_{1}, l_{1(m)}\right)+\sum_{i=1}^{j_{l}} \sum_{a=0}^{j_{l}-1} \mathcal{B}_{6}^{l} \mathcal{N}_{m, i, a}^{l} t^{a} f\left(k_{1}, l_{1(m, i)}\right)\right) .
\end{aligned}
$$


The integrations over $k_{1}$ and $l_{1}$ and the integration over $z$ can be combined in the closed form expression for the convergent part $\mathcal{V}_{\alpha}^{9-x}$ of the master integral $\mathcal{T}_{\alpha}^{9-x}$ in case of $\kappa_{i}, \lambda_{i} \rightarrow 0$. One has

$$
\begin{aligned}
\mathcal{V}_{\alpha}^{9-x}=\int \frac{\left(k_{0} \mp k_{1}\right)^{\alpha}\left(l_{0} \mp l_{1}\right)^{\beta}}{P_{1} P_{2} P_{3} P_{x} P_{6}} \mathcal{K}^{\left(j_{k}\right)} \mathcal{K}^{\left(j_{l}\right)} d^{4} k d^{4} l=\pi^{2} \int_{0}^{\infty} k_{0}^{\prime \alpha} d k_{0}^{\prime} l_{0}^{\beta} d l_{0}^{\prime} \int_{0}^{\infty} d s d t \\
\times \sum_{n=1}^{2} \sum_{m=x, 6}\left\{\frac{\mathcal{B}_{n, m}^{k l} \mathcal{N}_{n}^{k} \mathcal{N}_{m}^{l}}{\left(s-s_{1,2}-i \eta\right)\left(t-t_{x, 6}-i \eta\right) \sqrt[\star]{\left(A_{n, m}+i \eta\right)^{2}-4 s t}}\right. \\
\quad+\sum_{i=1}^{j_{k}} \sum_{a=0}^{2 j_{k}-2} \frac{\mathcal{B}_{0, m}^{k l} \mathcal{N}_{n, i, a}^{k} s^{a} \mathcal{N}_{m}^{l}}{\left(t-t_{x, 6}-i \eta\right) \sqrt[\star]{\left(A_{n, i, m}+i \eta\right)^{2}-4 s t}} \\
+\sum_{j=1}^{j_{l}} \sum_{b=0}^{j_{l}-1} \frac{\mathcal{B}_{n, 6}^{k l} \mathcal{N}_{n}^{k} \mathcal{N}_{m, j, b}^{l} t^{b}}{\left(s-s_{1,2}-i \eta\right) \sqrt[\star]{\left(A_{n, m, j}+i \eta\right)^{2}-4 s t}} \\
\left.+\sum_{i=1}^{j_{k}} \sum_{j=1}^{j_{l}} \sum_{a=0}^{2 j_{k}-2} \sum_{b=0}^{j_{l}-1} \frac{\mathcal{B}_{0,6}^{k l} \mathcal{N}_{n, i, a}^{k} s^{a} \mathcal{N}_{m, j, b}^{l} t^{b}}{\sqrt[\star]{\left(A_{n, i, m, j}+i \eta\right)^{2}-4 s t}}\right\}
\end{aligned}
$$

For the topology $\mathcal{T}^{4}(x=5), \mathcal{B}_{n, m}^{k l}$ is given by Eq. (68), i.e. it is the same as for the non-reduced planar topology. For the topology $\mathcal{T}^{5}(x=4)$, however, we obtain

$$
\mathcal{B}_{n, m}^{k l}=\mathcal{B}_{n}^{k} \mathcal{B}_{m}^{l}=-4 \pi^{2}\left[\theta\left(-\left(k_{0}^{\prime}+l_{0}^{\prime}\right)\right) \theta\left(-\theta_{n}\right) \theta\left(-\phi_{m}\right)+\theta\left(k_{0}^{\prime}+l_{0}^{\prime}\right) \theta\left(\theta_{n}\right) \theta\left(\phi_{m}\right)\right]
$$

The coefficients in the square roots read $A_{n, m}=A\left(k_{1(n)}, l_{1(m)}\right), A_{n, i, m}=A\left(k_{1(n, i)}, l_{1(m)}\right)$, $A_{n, m, j}=A\left(k_{1(n)}, l_{1(m, j)}\right)$, and $A_{n, i, m, j}=A\left(k_{1(n, i)}, l_{(m, j)}\right)$.

\subsection{Integral basis for the orthogonal space quadrature}

The integrals over $s$ and $t$ resulting for both the non-reduced and the rotated reduced planar topology are called basic integrals. They are of the types

$$
\begin{aligned}
\mathcal{F}\left(r_{s}, r_{t}, r_{0}, s_{0}, t_{0}\right) & =\int_{0}^{\infty} \frac{d s d t}{\left(s-s_{0}-i \eta\right)\left(t-t_{0}-i \eta\right) \sqrt[\star]{\left(r_{0}-r_{s} s-r_{t} t\right)^{2}-4 s t}}, \\
\mathcal{F}_{\alpha}^{s}\left(r_{s}, r_{t}, r_{0}, t_{0}\right) & =\int_{0}^{\infty} \frac{s^{\alpha} d s d t}{\left(t-t_{0}-i \eta\right) \sqrt[\star]{\left(r_{0}-r_{s} s-r_{t} t\right)^{2}-4 s t}} \\
\mathcal{F}_{\beta}^{t}\left(r_{s}, r_{t}, r_{0}, s_{0}\right) & =\int_{0}^{\infty} \frac{t^{\beta} d s d t}{\left(s-s_{0}-i \eta\right) \sqrt[\star]{\left(r_{0}-r_{s} s-r_{t} t\right)^{2}-4 s t}} \\
\mathcal{F}_{\alpha, \beta}^{s t}\left(r_{s}, r_{t}, r_{0}\right) & =\int_{0}^{\infty} \frac{s^{\alpha} t^{\beta} d s d t}{\sqrt[\star]{\left(r_{0}-r_{s} s-r_{t} t\right)^{2}-4 s t}} .
\end{aligned}
$$


All these integrals are divergent except for the first one. However, after the subtraction they occur in sums in which the divergences vanish. While the integral $\mathcal{F}\left(r_{s}, r_{t}, r_{0}, s_{0}, t_{0}\right)$ is calculated in Refs. [35, 54, 58, the other integrals are new. The results can be found in Appendix $\mathrm{A}$ in terms of the parameters $r_{s}, r_{t}, r_{0}, s_{0}$, and $t_{0}$. In this paragraph we are dealing only with the dependence on the parameters $r_{s}, r_{t}$, and $r_{0}$ and on the poles found in the residue integration. In case of the integral $\mathcal{F}\left(r_{s}, r_{t}, r_{0}, s_{0}, t_{0}\right)$ we obtain

$$
\begin{aligned}
& r_{0}=\left(k_{0}^{\prime}+l_{0}^{\prime}\right)^{2} \mp 2\left(\frac{\xi_{n}+i \eta}{\theta_{n}}+\frac{\psi_{m}+i \eta}{\phi_{m}}\right)\left(k_{0}^{\prime}+l_{0}^{\prime}\right)-m_{3}^{2}-i \eta=: r_{n, m}, \\
& r_{s}=1 \mp 2 \frac{k_{0}^{\prime}+l_{0}^{\prime}}{\theta_{n}}, \quad r_{t}=1 \mp 2 \frac{k_{0}^{\prime}+l_{0}^{\prime}}{\phi_{m}}
\end{aligned}
$$

where the signs correspond to the two linearizations $l_{0}=l_{0}^{\prime} \pm l_{1}$. Note that $r_{s}, r_{t}>0$ and $r_{s} r_{t}>1$ in regions where the numerical integration has to be done. For $\mathcal{F}_{\alpha}^{s}$ we have to replace $\theta_{n}$ by $\theta_{0}$ and $\xi_{n}$ by $\xi_{n, i}$. For $\mathcal{F}_{\beta}^{t}$ we have to replace $\phi_{m}$ by $\phi_{6}$ and $\psi_{m}$ by $\psi_{m, j}$. For $\mathcal{F}_{\alpha, \beta}^{s t}$, finally, both replacements have to be performed. Note that up to the choice for the parameters $s_{0}, t_{0}, r_{s}$ and $r_{t}, \mathcal{F}_{\alpha}^{s}$ and $\mathcal{F}_{\beta}^{t}$ are the same integrals.

The imaginary parts in the rational factors in the numerator are used to separate real and imaginary parts according to the Sokhotsky-Plemely relations

$$
\lim _{\eta \rightarrow+0} \int_{a}^{b} \frac{f(x) d x}{x-x_{0} \pm i \eta}=\mathrm{P} \int_{a}^{b} \frac{f(x) d x}{x-x_{0}} \mp i \pi \int_{a}^{b} \delta\left(x-x_{0}\right) f(x) d x
$$

where "P" indicates the principal value integral.

\subsection{Analytic behaviour of the modified square root}

We have to consider different cases in order to analyze the analytic behaviour of the square root occurring in the integrand [34, 35, 54]. The equation

$$
R(s, t)=\left(r_{0}-r_{s} s-r_{t} t\right)^{2}-4 s t=0
$$

parametrizes an ellipse which separates the positive and negative values of the radicand. Outside of the ellipse the radicand is positive, while for points inside the ellipse it takes negative values. The ellipse touches the axes at $s=r_{0} / r_{s}$ and $t=r_{0} / r_{t}$. For $r_{0}<0$, therefore, the ellipse is located in the third quadrant. But because the integration is performed in the first quadrant $(s \in[0, \infty], t \in[0, \infty])$, the square root will give real values and no imaginary part is produced. For $r_{0}>0$, however, the ellipse moves into the integration region and imaginary parts enter the calculations. 


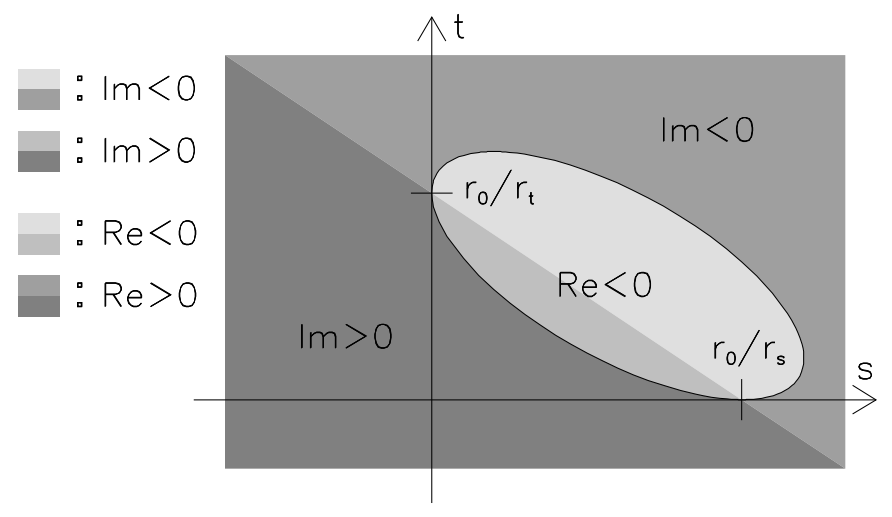

Figure 2: Regions in the $(s, t)$-plane with different real and imaginary parts for the radicand $R(s, t)=\left(r_{0}-r_{s} s-r_{t} t\right)^{2}-4 s t$

In order to evaluate the square root uniquely, we also consider the imaginary part of the radicand, caused by the term $i \eta$. It turns out that the sign of the imaginary part is determined by the sign of $r_{0}-r_{s} s-r_{t} t$, the zeros of which are given by the straight line connecting the points $s=r_{0} / r_{s}$ and $t=r_{0} / r_{t}$ on the axes where the ellipse touches the axes. Above this line (as seen from the origin for $r_{0}>0$ ), the imaginary part is negative, below this line it is positive. The situation is shown in Fig. 2

We distinguish the following cases:

- outside the ellipse and below the line $(\operatorname{Re} R(s, t)>0, \operatorname{Im} R(s, t)>0)$ we have

$$
\sqrt[\star]{\left(r_{0}-r_{s} s-r_{t} t\right)^{2}-4 s t}=+\sqrt{\left(r_{0}-r_{s} s-r_{t} t\right)^{2}-4 s t}
$$

- outside the ellipse and above the line $(\operatorname{Re} R(s, t)>0, \operatorname{Im} R(s, t)<0)$ we have

$$
\sqrt[\star]{\left(r_{0}-r_{s} s-r_{t} t\right)^{2}-4 s t}=-\sqrt{\left(r_{0}-r_{s} s-r_{t} t\right)^{2}-4 s t}
$$

- inside the ellipse $(\operatorname{Re} R(s, t)<0)$ we have

$$
\sqrt[\star]{\left(r_{0}-r_{s} s-r_{t} t\right)^{2}-4 s t}=i \sqrt{4 s t-\left(r_{0}-r_{s} s-r_{t} t\right)^{2}}
$$

\section{Landau singularities and thresholds}

Multiloop integrals with many propagator factors are influenced by their singularity structure. The pole structure can be analyzed by using Feynman parametrization [59, 60]. 
Starting from

$$
F\left(p_{j}, m_{k}\right)=\int\left(\prod_{l=1}^{L} d^{4} k_{l}\right) f\left(p_{j}, k_{l}, m_{k}\right) \prod_{n=1}^{N} \frac{i}{A_{n}}, \quad A_{n}=q_{n}^{2}-m_{n}^{2}+i \eta
$$

with $E$ outer momenta $p_{j}, L$ loop momenta $k_{l}, N$ inner masses $m_{n}$, and $q_{n}$ a linear combination of $p_{j}$ and $k_{l}$, we can apply the Feynman parametrization

$$
\frac{1}{A_{1} A_{2} \cdots A_{N}}=(N-1) ! \int_{0}^{1} \frac{\delta\left(\lambda_{1}+\lambda_{2}+\ldots+\lambda_{N}-1\right) d \lambda_{1} d \lambda_{2} \cdots d \lambda_{N}}{\left(\lambda_{1} A_{1}+\lambda_{2} A_{2}+\ldots+\lambda_{N} A_{N}\right)^{N}} .
$$

By shifting the inner momenta $k_{l}$ to $k_{l}^{\prime}$ by finite amounts (which are determined by the outer momenta), the denominator can be rewritten as

$$
\mathcal{D}=\sum_{n=1}^{N} \lambda_{n} A_{n}=\phi+K\left(k_{1}^{\prime}, k_{2}^{\prime}, \ldots, k_{L}^{\prime}\right)
$$

where $\phi$ is independent of inner momenta and $K$ is a quadratic form in the shifted momenta $k_{l}^{\prime}$. It can then be shown [59, 60] that the denominator vanishes, i.e. the integral has socalled Landau singularities, if

$$
\begin{aligned}
\lambda_{n}\left(q_{n}^{2}-m_{n}^{2}\right) & =0 & \text { for all } n=1, \ldots N \text { (first Landau equation) } \\
\text { and } \sum_{n=1}^{N} \lambda_{n} q_{n} & =0 & \text { for each loop (second Landau equation). }
\end{aligned}
$$
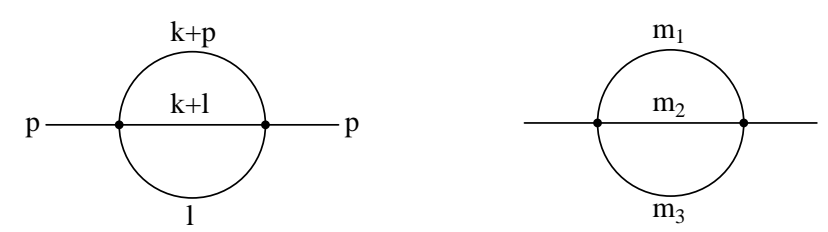

Figure 3: Sunset diagram with momenta (left hand side) and masses (right hand side)

\subsection{Two- and three-particle thresholds}

The Landau equations can be used to analyze a given Feynman diagram. For two-point functions, singularities occur if the squared outer momentum crosses thresholds or pseudothresholds. For a genuine sunset diagram as shown in Fig. 3, a two-loop two-point 
function with three lines of mass values $m_{1}, m_{2}$ and $m_{3}$ connecting the incoming with the outgoing leg, these thresholds and pseudothresholds are given by

$$
p^{2}=\left(m_{1} \pm m_{2} \pm m_{3}\right)^{2}
$$

where the three-particle threshold is located at $p^{2}=\left(m_{1}+m_{2}+m_{3}\right)^{2}$. In general, the genuine threshold is the one with the highest value. If the squared momentum crosses this threshold, an imaginary part appears. According to the Cutkosky rules [61], this imaginary part corresponds to the situation where the particles move onto their mass shell. In terms of Feynman diagrams, inner lines are cut into outer legs for physical particles. This can be visualized in Feynman diagrams by drawing a cutting line.

In the diagrams we are working with we expect two- and three-particle thresholds. While the two-particle thresholds are related to the values of $s_{0}=s_{n, m}$ and $t_{0}=t_{n, m}$, all the three-particle thresholds are related to the values of $r_{0}=r_{n, m}$,

$$
\begin{array}{rlllll}
s_{1,2}>0 & \Leftrightarrow & p^{2}>\left(m_{1}+m_{2}\right)^{2}, & r_{1,5}>0 & \Leftrightarrow & p^{2}>\left(m_{1}+m_{3}+m_{5}\right)^{2}, \\
t_{4,5}>0 & \Leftrightarrow & p^{2}>\left(m_{4}+m_{5}\right)^{2}, & r_{2,4}>0 & \Leftrightarrow & p^{2}>\left(m_{2}+m_{3}+m_{4}\right)^{2}, \\
t_{4,6}>0 & \Leftrightarrow & p^{2}>\left(m_{4}+m_{6}\right)^{2}, & r_{1,6}>0 & \Leftrightarrow & p_{1}^{2}>\left(m_{1}+m_{3}+m_{6}\right)^{2}, \\
t_{5,6}>0 & \Leftrightarrow & p^{2}>\left(m_{5}+m_{6}\right)^{2}, & r_{2,6}>0 & \Leftrightarrow & p_{2}^{2}>\left(m_{2}+m_{3}+m_{6}\right)^{2} .
\end{array}
$$

Anticipating examples to follow, for the subtraction terms some of the two-particle thresholds vanish while new three-particle thresholds appear. For the diagram shown on the right hand side of Fig. 51, additional three-particle thresholds appear for $p_{1}^{2}>\left(m_{n, j}+m_{3}+m_{4}\right)^{2}$ $\left(r_{n, j, 4}>0\right)$ and $p_{2}^{2}>\left(m_{n, j}+m_{3}+m_{5}\right)^{2}\left(r_{n, j, 5}>0\right)$ while two-particle thresholds including the masses $m_{1}$ and $m_{2}$ no longer occur.

\subsection{Landau singularities for the scalar topology}

As an example we consider the original planar topology in Eq. (42) with $\alpha=0$. Because we are only interested in a qualitative analysis, we use the following fictitious values for masses and outgoing momenta,

$$
\begin{array}{cccc}
m_{1}=420 \mathrm{GeV} & m_{3}=100 \mathrm{GeV} & m_{4}=120 \mathrm{GeV} & \sqrt{p_{1}^{2}}=60 \mathrm{GeV} \\
m_{2}=80 \mathrm{GeV} & m_{5}=200 \mathrm{GeV} & m_{6}=300 \mathrm{GeV} & \sqrt{p_{2}^{2}}=20 \mathrm{GeV}
\end{array}
$$

For the decaying particle we vary the value of $M=\sqrt{p^{2}}$ in a range between $M=100 \mathrm{GeV}$ and $M=800 \mathrm{GeV}$ and plot the real and imaginary part. The result is shown in Fig. 4 . 

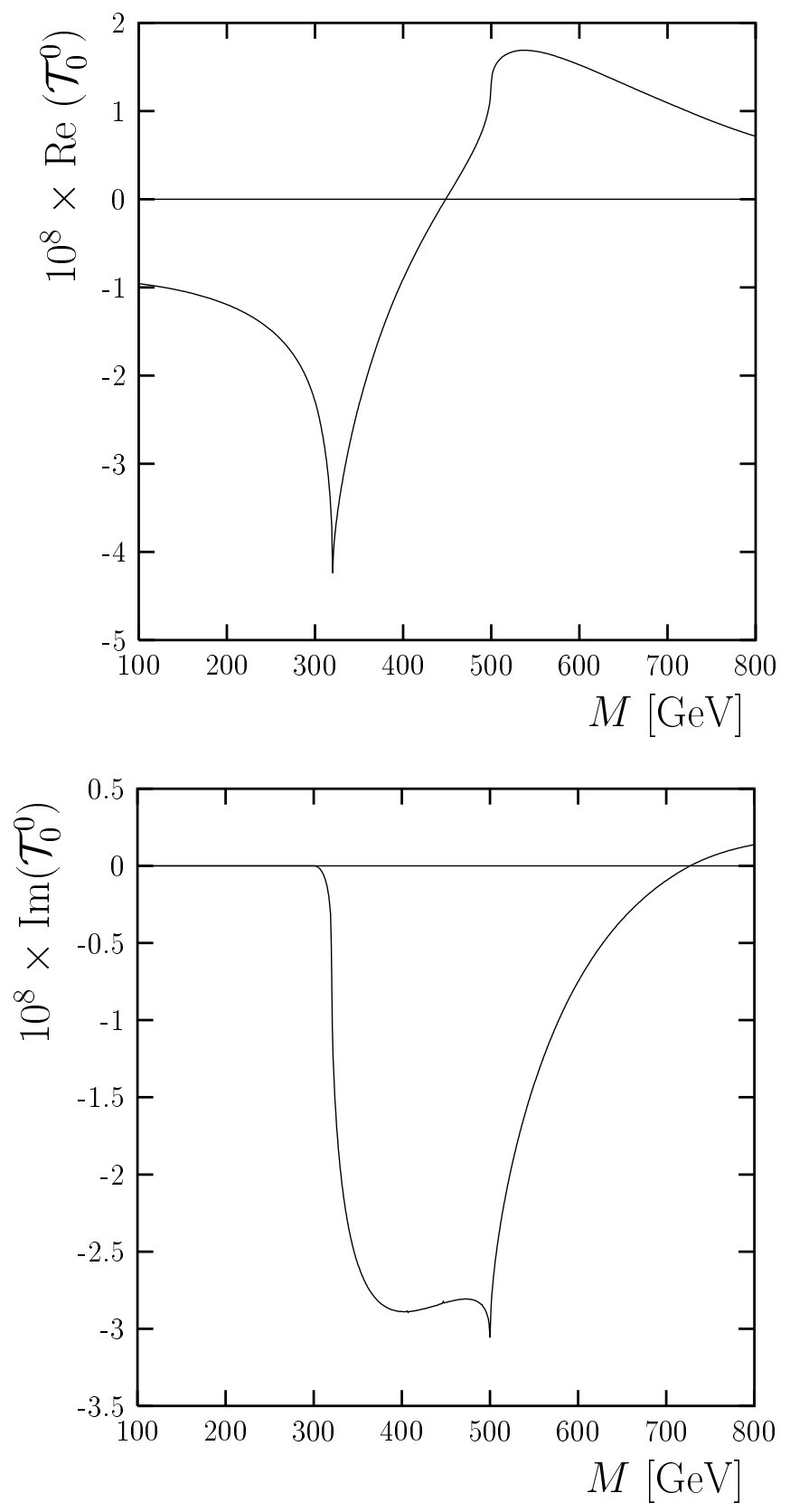

Figure 4: Real part (top) and and imaginary part (bottom) for the scalar planar two-loop three-point diagram $\mathcal{T}_{0}^{0}$ of Eq. (42) with decay mass $M$ between $100 \mathrm{GeV}$ and $800 \mathrm{GeV}$. For the values of masses and momenta we use the standard set given in Eq. (101). 
A three-particle threshold is expected for $M=m_{2}+m_{3}+m_{4}=300 \mathrm{GeV}$. At this point we see that the imaginary part starts to differ from zero. The two-particle threshold at $M=m_{4}+m_{5}=320 \mathrm{GeV}$ is characterized by a sharp peak in the real part, accompanied by an instant decrease of the imaginary part, leading to a vertical tangent to the curve at this point. For the second two-particle threshold in this energy range at $M=m_{1}+m_{2}=$ $500 \mathrm{GeV}$, the situation is the opposite. The imaginary part shows a sharp peak while the real part increases with vertical slope.

\subsection{The topology of the subtraction terms}

If we consider tensor integrals we have to take subtraction terms into account. As it was shown in the previous section, for the planar two-loop three-point master diagram with power $\left(k_{0}-k_{1}\right)^{2}$ we need a single subtraction. Each subtraction replaces the propagator factors by the subtracted ones. In the present case one has

$$
\mathcal{V}_{2}^{0}=\int \frac{\left(k_{0}-k_{1}\right)^{2} d^{4} k d^{4} l}{P_{1} P_{2} P_{3} P_{4} P_{5} P_{6}}\left(1-\frac{P_{1} P_{2}}{P_{1,1} P_{2,1}}\right)=\int \frac{\left(k_{0}-k_{1}\right)^{2} d^{4} k d^{4} l}{P_{1} P_{2} P_{3} P_{4} P_{5} P_{6}}-\int \frac{\left(k_{0}-k_{1}\right)^{2} d^{4} k d^{4} l}{P_{1,1} P_{2,1} P_{3} P_{4} P_{5} P_{6}}
$$

(for higher order subtractions the scaled propagator factors appear in higher powers). If we perform the limit $\kappa \rightarrow 0$ for $P_{1,1}=\left(k+\kappa p_{1}\right)^{2}-m_{1,1}^{2}-i \eta$ and $P_{2,1}=\left(k-\kappa p_{2}\right)^{2}-m_{2,1}^{2}-i \eta$, the propagator factors loose their dependence on the outer momenta $p_{i}$. In this case the momentum scheme given for the original diagram is no longer valid. We first have to perform a partial fraction decomposition for the subtraction term,

$$
\int \frac{\left(k_{0}-k_{1}\right)^{2} d^{D} k d^{D} l}{P_{1,1} P_{2,1} P_{3} P_{4} P_{5} P_{6}}=\frac{1}{m_{2,1}^{2}-m_{1,1}^{2}}\left[\int \frac{\left(k_{0}-k_{1}\right)^{2} d^{D} k d^{D} l}{P_{1,1} P_{3} P_{4} P_{5} P_{6}}-\int \frac{\left(k_{0}-k_{1}\right)^{2} d^{D} k d^{D} l}{P_{2,1} P_{3} P_{4} P_{5} P_{6}}\right] .
$$

After that, the lines can be rearranged according to their momenta. The planar diagram is shown in Fig. 5 together with the subtraction. For convenience, relative factors are not mentioned in the diagrammatic representation.

\section{Numerical integration}

After having performed all other integrations analytically, only the two integrations over $k_{0}^{\prime}$ and $l_{0}^{\prime}$ are left. The integration region is given by the non-vanishing of the factors $\mathcal{B}_{n, m}^{k l}$ 


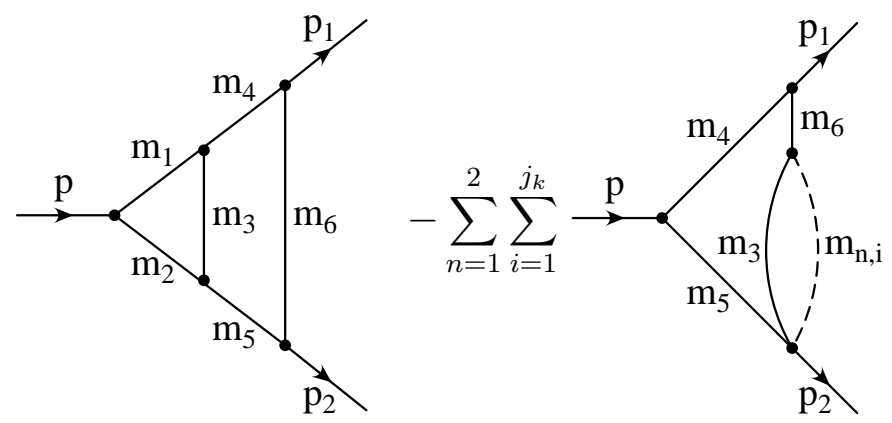

Figure 5: Planar topology $\mathcal{T}^{0}$ (left hand side) and the subtraction terms (right hand side). For convenience, relative factors are not mentioned in the diagrammatic representation. The numerator factor for the master integral and the subtraction terms read $\left(k_{0}-k_{1}\right)^{\alpha}$.

which read

$$
\mathcal{B}_{n, m}^{k l}=-4 \pi^{2}\left[\theta\left( \pm\left(k_{0}^{\prime}+l_{0}^{\prime}\right)\right) \theta\left(-\theta_{n}\right) \theta\left(-\phi_{m}\right)+\theta\left( \pm\left(k_{0}^{\prime}+l_{0}^{\prime}\right)\right) \theta\left(\theta_{n}\right) \theta\left(\phi_{m}\right)\right]
$$

depending on the linearizations $k_{0}=k_{0}^{\prime} \pm k_{1}$ and $l_{0}=l_{0}^{\prime} \pm l_{1}$. If we take into account that

$$
\begin{array}{lll}
\theta_{1}= \pm 2\left(k_{0}^{\prime}+E_{1} \mp q_{z}\right), & \theta_{2}= \pm 2\left(k_{0}^{\prime}-E_{2} \mp q_{z}\right), & \theta_{0}= \pm 2 k_{0}^{\prime} \\
\phi_{4}= \pm 2\left(l_{0}^{\prime}-E_{1} \pm q_{z}\right), & \phi_{5}= \pm 2\left(l_{0}^{\prime}+E_{2} \pm q_{z}\right), & \phi_{6}= \pm 2 l_{0}^{\prime},
\end{array}
$$

the integration regions can be seen to be triangles which are bound by the off-diagonal $k_{0}^{\prime}+l_{0}^{\prime}=0$ and the different unequalities coming from $\theta_{n}$ and $\phi_{m}$. In case of the index couples $(n, m)=(1,4),(2,5),(0,6)$ the integration region vanishes identically. The nonvanishing integration regions are shown in Fig. 6] in case of the linearizations $k_{0}=k_{0}^{\prime}+k_{1}$ and $l_{0}=l_{0}^{\prime}+l_{1}$. Two remarks are in order at this point.

- Special care has to be taken for the case $(n, m)=(0,6)$. In this case, the integration region vanishes only in the limit $\kappa_{i}, \lambda_{i} \rightarrow 0$. However, we have thoroughly checked that in this limit the integrand does not diverge, i.e. the integral does not give a non-vanishing contribution even with a vanishing integration region 62 .

- In case that one of the masses vanishes, integration regions might vanish. In Fig. 6] we see that if $E_{1}=q_{z}$, i.e. if $p_{1}^{2}=0$, the triangles $(n, m)=(1,6),(0,4)$ vanish. In case of the opposite linearization and with $p_{2}^{2}=0$, the same holds for $(n, m)=(2,6),(0,5)$ instead. However, the integrand does not diverge in these cases. 


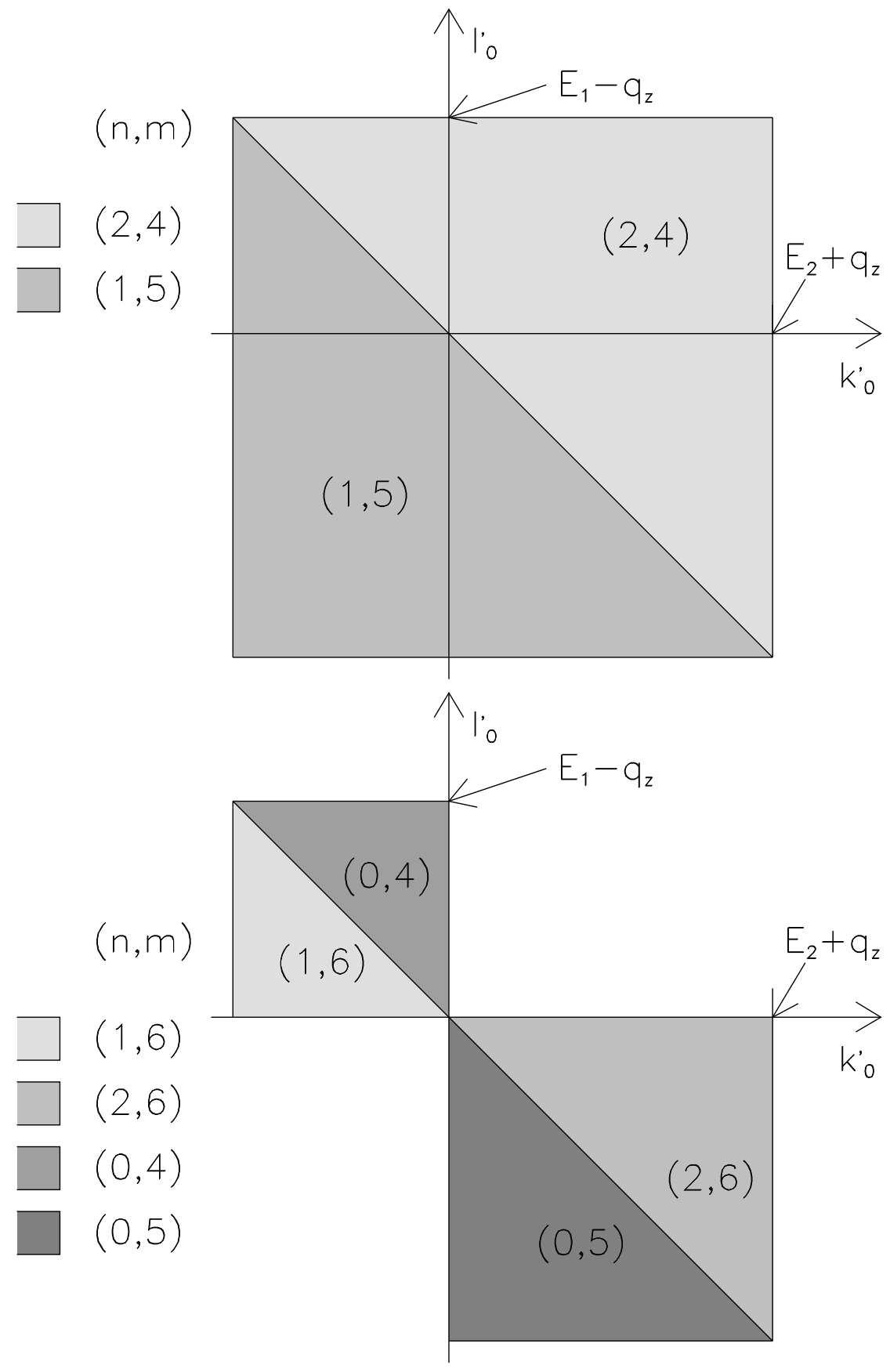

Figure 6: The integration regions for the last (numerical) two-dimensional integration are triangles which are bound by the line $k_{0}^{\prime}+l_{0}^{\prime}=0$, a vertical and a horizontal line, according to the condition that $\mathcal{B}_{n, m}^{k l}$ in Eqs. (68) or (899) does not vanish. The integration regions for different values of index couples $(n, m)$ are shown for the linearizations $k_{0}=k_{0}^{\prime}+k_{1}$ and $l_{0}=l_{0}^{\prime}+l_{1}$. In case of the opposite linearizations, the signs in front of the $q_{z}$ change. 


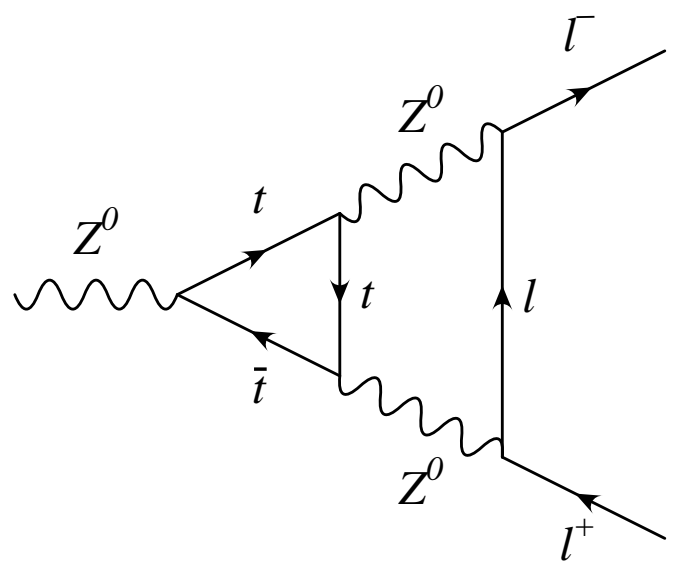

Figure 7: Feynman diagram corresponding to $\mathcal{V}_{2}^{0}$ in Eq. (102)

The integrations are performed using numerical routines like the Monte Carlo integration routine VEGAS [43, 44] and the program Divonne of the library CUBA [45]. While numerical routines sometimes overestimate the precision of the result [63, 64, the use of two different routines enables us to have a measure for the accuracy of the result. In this section we will present a few examples in order to demonstrate the reliability of our method.

\subsection{The $Z^{0}$ decay via top quark loop}

As a first example we consider the basic integral in Eq. (102) occuring in a process where the $Z^{0}$ boson couples to a triangle with top quark current (cf. Fig. 7). We have started the discussion already in Eq. (102) and will continue at this point. The masses are chosen to be

$$
m_{1}=m_{2}=m_{3}=m_{t}, \quad m_{4}=m_{5}=m_{Z}, \quad \text { and } \quad m_{6}=m_{l}
$$

where we use the values $m_{t}=178 \mathrm{GeV}, m_{Z}=91.1876 \mathrm{GeV}[65]$, and $m_{l}=0$. The subtraction masses which should have different values are taken as $m_{1,1}=150 \mathrm{GeV}$ and $m_{2,1}=160 \mathrm{GeV}$. Because the squared mass of the $Z^{0}$ boson is below all possible thresholds, the imaginary part of this integral vanishes. Even though physical masses are chosen in this paragraph, we stress that instead of calculating the whole process we are dealing with the occuring planar basic diagram only.

We calculate the integral for 50 points between $\kappa_{1}=0.01$ and $\kappa_{1}=0.5$ for the (dimensionless) parameter $\kappa_{1}$. As a next step we approximate the points by a low degree 


\begin{tabular}{|r|lll|}
\hline degree & real part & $\sigma_{\mathrm{Re}}$ & $\chi_{\mathrm{Re}}^{2} /(n-1)$ \\
\hline 0 & $-2.941141 \cdot 10^{-4}$ & $1.4 \cdot 10^{-9}$ & $1.2 \cdot 10^{7}$ \\
1 & $-3.484637 \cdot 10^{-4}$ & $2.6 \cdot 10^{-9}$ & $3.8 \cdot 10^{5}$ \\
2 & $-3.361064 \cdot 10^{-4}$ & $3.9 \cdot 10^{-9}$ & $1.4 \cdot 10^{1}$ \\
3 & $-3.361950 \cdot 10^{-4}$ & $5.2 \cdot 10^{-9}$ & $1.4 \cdot 10^{0}$ \\
4 & $-3.361836 \cdot 10^{-4}$ & $6.8 \cdot 10^{-9}$ & $1.3 \cdot 10^{0}$ \\
5 & $-3.361884 \cdot 10^{-4}$ & $8.6 \cdot 10^{-9}$ & $1.3 \cdot 10^{0}$ \\
6 & $-3.361693 \cdot 10^{-4}$ & $1.1 \cdot 10^{-8}$ & $1.1 \cdot 10^{0}$ \\
\hline $\mathrm{x}$ & $-3.365555 \cdot 10^{-4}$ & $7.2 \cdot 10^{-9}$ & \\
\hline
\end{tabular}

Table 1: Extrapolation of the contributions for $\mathcal{V}_{2}^{0}$ using VEGAS

\begin{tabular}{|r|lll|}
\hline degree & real part & $\sigma_{\mathrm{Re}}$ & $\chi_{\mathrm{Re}}^{2} /(n-1)$ \\
\hline 0 & $-2.81249 \cdot 10^{-4}$ & $3.9 \cdot 10^{-8}$ & $1.7 \cdot 10^{4}$ \\
1 & $-3.52842 \cdot 10^{-4}$ & $8.7 \cdot 10^{-8}$ & $5.0 \cdot 10^{2}$ \\
2 & $-3.36111 \cdot 10^{-4}$ & $1.3 \cdot 10^{-7}$ & $1.0 \cdot 10^{-1}$ \\
3 & $-3.36265 \cdot 10^{-4}$ & $1.9 \cdot 10^{-7}$ & $7.4 \cdot 10^{-2}$ \\
4 & $-3.36301 \cdot 10^{-4}$ & $2.5 \cdot 10^{-7}$ & $7.3 \cdot 10^{-2}$ \\
5 & $-3.36294 \cdot 10^{-4}$ & $3.2 \cdot 10^{-7}$ & $7.3 \cdot 10^{-2}$ \\
6 & $-3.36209 \cdot 10^{-4}$ & $4.1 \cdot 10^{-7}$ & $7.1 \cdot 10^{-2}$ \\
\hline $\mathrm{x}$ & $-3.36530 \cdot 10^{-4}$ & $3.3 \cdot 10^{-7}$ & \\
\hline
\end{tabular}

Table 2: Extrapolation of the contributions for $\mathcal{V}_{2}^{0}$ using Divonne

polynomial in order to extrapolate to $\kappa_{1}=0$. The result of this extrapolation can then be compared with the result for the integral where we used $\kappa_{1}=0$ from the very beginning. For the integration with VEGAS, 260000 internal points were used, whereas for Divonne we use a standard precision of $10^{-3}$. The results of this fit are shown in Tables 1 and 2. If $\kappa_{1}=0$ is used from the very beginning and all integrations are done analytically except for the last two ones, we obtain the results shown in the last lines of Tables 1 and 2 marked by a " $\mathrm{x}$ ". Obviously, these values are already reliably approximated by the second order fit. In case of VEGAS, the quantity $\chi^{2} /(n-1)$ for $n$ sampling points as a measure for the reliability of the polynomial fit of the numerical integrations approaches the optimal value 1.0 quite fast. The deviation of the order of 1 in case of Divonne is not problematic because we use this second method only to check the VEGAS calculation. The result is shown graphically in Fig. 8. In this figure we show the results of the numerical calculation as a function of $\kappa_{1}$ together with the result for the semi-analytical calculation for $\kappa_{1}=0$. 


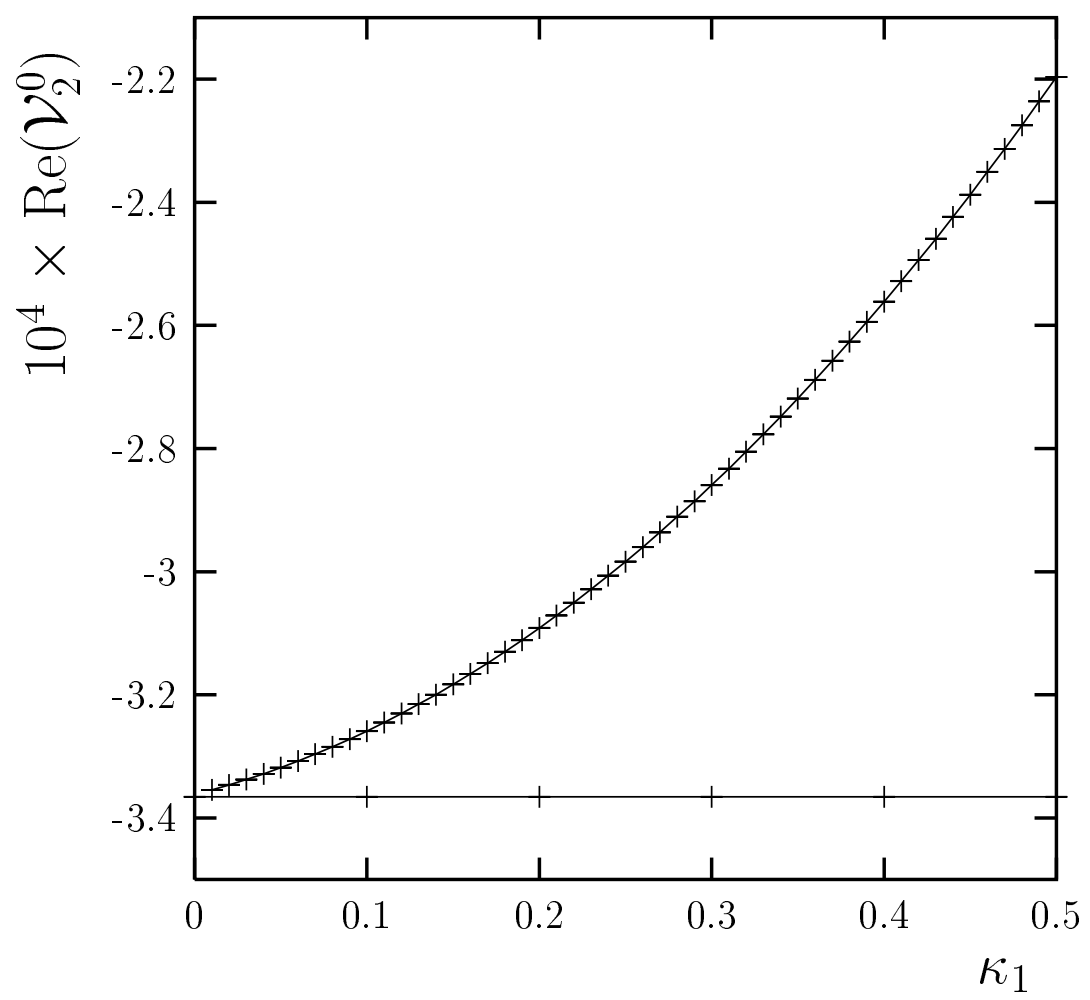

Figure 8: Real part of the integral $\mathcal{V}_{2}^{0}$ in Eq. (102) as a function of the parameter $\kappa_{1}$. For the masses and momenta we used values related to the decay of the $Z^{0}$ boson into a top-quark loop, as explained in the text (cf. Fig. 7). Shown are the results for the numerical calculation (curve) and the result for the semi-analytical calculation with $\kappa_{1}=0$ (horizontal straight line). In both cases we have used VEGAS. 


\begin{tabular}{|r|lll|lll|}
\hline degree & real part & $\sigma_{\mathrm{Re}}$ & $\chi_{\mathrm{Re}}^{2} /(n-1)$ & imag. part & $\sigma_{\mathrm{Im}}$ & $\chi_{\mathrm{Im}}^{2} /(n-1)$ \\
\hline 2 & $2.71015 \cdot 10^{3}$ & $3.0 \cdot 10^{-1}$ & $8.5 \cdot 10^{-1}$ & $6.67079 \cdot 10^{3}$ & $7.7 \cdot 10^{-1}$ & $1.1 \cdot 10^{0}$ \\
3 & $2.71047 \cdot 10^{3}$ & $4.2 \cdot 10^{-1}$ & $8.3 \cdot 10^{-1}$ & $6.6712 \cdot 10^{3}$ & $1.1 \cdot 10^{0}$ & $1.1 \cdot 10^{0}$ \\
\hline $\mathrm{x}$ & $2.71125 \cdot 10^{3}$ & $7.0 \cdot 10^{-1}$ & & $6.6703 \cdot 10^{3}$ & $1.7 \cdot 10^{0}$ & \\
\hline
\end{tabular}

Table 3: Extrapolation of the contributions for $\mathcal{V}_{21}^{5}$ using VEGAS

\begin{tabular}{|r|lll|lll|}
\hline degree & real part & $\sigma_{\mathrm{Re}}$ & $\chi_{\mathrm{Re}}^{2} /(n-1)$ & imag. part & $\sigma_{\operatorname{Im}}$ & $\chi_{\operatorname{Im}}^{2} /(n-1)$ \\
\hline 2 & $2.7094 \cdot 10^{3}$ & $1.0 \cdot 10^{0}$ & $4.4 \cdot 10^{-1}$ & $6.6691 \cdot 10^{3}$ & $2.7 \cdot 10^{0}$ & $3.7 \cdot 10^{-1}$ \\
\hline $\mathrm{x}$ & $2.7042 \cdot 10^{3}$ & $2.6 \cdot 10^{0}$ & & $6.6736 \cdot 10^{3}$ & $6.6 \cdot 10^{0}$ & \\
\hline
\end{tabular}

Table 4: Extrapolation of the contributions for $\mathcal{V}_{21}^{5}$ using Divonne

The error bars for the latter are plotted at different points in order to allow for a better comparison. Also optically the results for numerical and semi-analytical calculation match very well. This gives us confidence in the correct implementation of the algorithm.

\subsection{Rotated reduced planar topology}

Next we consider the numerical results for a specific example of the rotated reduced planar topology $\mathcal{T}^{5}$. The physical starting point is a decay of the $Z^{0}$ boson via a lepton loop, coupling to the outer lepton and anti-lepton legs via $W$ bosons. The integral

$$
\mathcal{V}_{21}^{5}=\int \frac{\left(k_{0}+k_{1}\right)^{2}\left(l_{0}+l_{1}\right)}{P_{1} P_{2} P_{3} P_{4} P_{6}}\left(1-\frac{P_{1} P_{2}}{P_{1,1} P_{2,1}}\right)\left(1-\frac{P_{4}}{P_{4,1}}\right) d^{4} k d^{4} l
$$

contains two subtraction terms, for the $k$-loop as well as for the $l$-loop. In this case we consider the limit $\kappa_{1}=0.8 \cdot \lambda_{1} \rightarrow 0$. The diagram with rotated reduced planar topology is shown in Fig. [10 together with the three different types of subtraction terms. The masses take the values $m_{1}=m_{2}=m_{3}=m_{l}=0, m_{4}=m_{W}=80.425 \mathrm{GeV}$ [65], and $m_{6}=m_{l}=0$. The momenta are given by $\sqrt{p_{1}^{2}}=\sqrt{p_{2}^{2}}=m_{l}=0$ and $\sqrt{p^{2}}=m_{Z}$. For the three subtraction masses we take $m_{1,1}=200 \mathrm{GeV}, m_{2,1}=300 \mathrm{GeV}$, and $m_{4,1}=110 \mathrm{GeV}$. In Table 3 we show the results for the numerical integration with VEGAS for the real and the imaginary part. Only the values at polynomial degrees 2 and 3 are shown. They are in good agreement with the result at polynomial degree 2 obtained by using Divonne (Table 4). The results for the semi-analytical calculation are again given in the last lines of the two tables. Looking at the values obtained by using VEGAS in Fig. 9, one sees good agreement. 

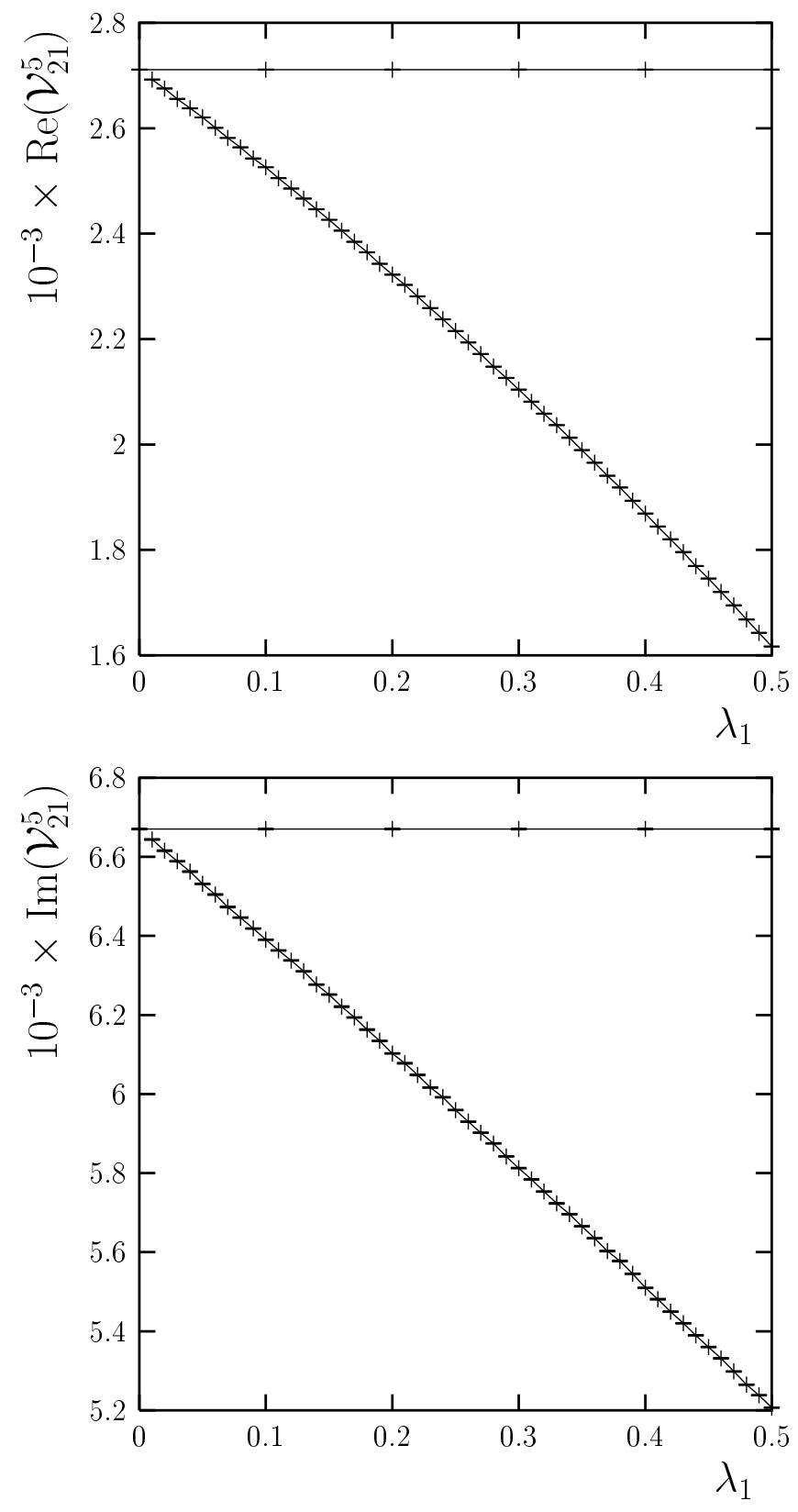

Figure 9: Real part (top) and imaginary part (bottom) of the integral $\mathcal{V}_{21}^{5}$ in Eq. (107) as a function of the parameters $\lambda_{1}$ and $\kappa_{1}=0.8 \cdot \lambda_{1}$. Shown are the results for the numerical calculation (curve) and for the semi-analytical calculation with $\kappa_{1}=\lambda_{1}=0$ (horizontal straight line), both obtained by using VEGAS. 


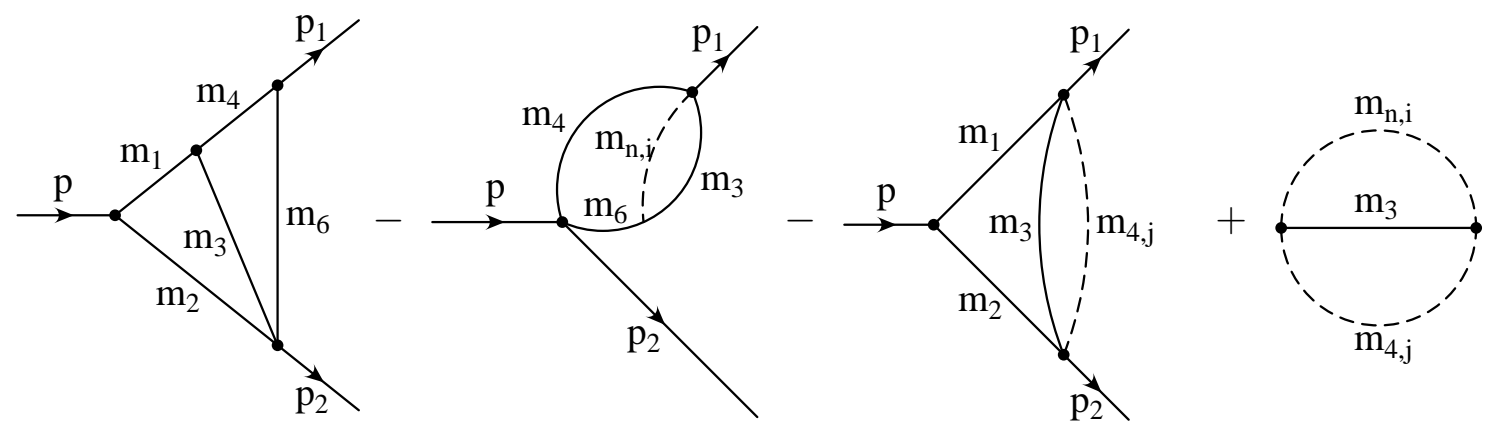

Figure 10: Rotated reduced planar topology $\mathcal{T}^{5}$ (first diagram) and the subtraction terms. For convenience, relative factors are not mentioned in the diagrammatic representation. Summations over $n=1,2, i=1, \ldots, j_{k}$ and $j=1, \ldots, j_{l}$ are assumed (cf. Fig. [5). The numerator factor for the master integral and the subtraction terms reads $\left(k_{0}+k_{1}\right)^{\alpha}\left(l_{0}+l_{1}\right)^{\beta}$.

\subsection{Results for varying decay mass}

For the integral with two subtractions for the $k$-loop,

$$
\mathcal{V}_{3}^{0}=\int \frac{\left(k_{0}-k_{1}\right)^{3} d^{4} k d^{4} l}{P_{1} P_{2} P_{3} P_{4} P_{5} P_{6}} \prod_{i=1}^{2}\left(1-\frac{P_{1} P_{2}}{P_{1, i} P_{2, i}}\right)
$$

we vary the decay mass $M=\sqrt{p^{2}}$ in order to identify different thresholds. The values for physical masses and outgoing momenta are again taken from Eq. (101), for the subtraction masses we use $m_{1,1}=100 \mathrm{GeV}, m_{2,1}=200 \mathrm{GeV}, m_{1,2}=350 \mathrm{GeV}$, and $m_{2,2}=450 \mathrm{GeV}$. In varying $M$ we can observe the behaviour of the real and the imaginary part. The behaviour is shown in Fig. 11 for decay mass values close to $M=320 \mathrm{GeV}$. As in case of the simple example shown in Fig. 4 we notice a sharp peak for the real part and a vertical slope for the imaginary part at the point $M=320 \mathrm{GeV}$ which corresponds to the two-particle threshold $M^{2}=p^{2}=\left(m_{4}+m_{5}\right)^{2}$. In a second step we are looking more closely at the threshold region. Taking the value $M=325 \mathrm{GeV}$, we analyze the real and imaginary parts for $\kappa_{2}=0.6 \cdot \kappa_{1} \rightarrow 0$. In Table 5 we show results of the polynomial fit for the results obtained by VEGAS. The last line of Table 5 and Table [6] are the semi-analytic results obtained by using VEGAS and Divonne, respectively. The results are shown in Fig. 12. Again both methods lead to the same result in the limit $\kappa_{2}=0.6 \cdot \kappa_{1} \rightarrow 0$. 

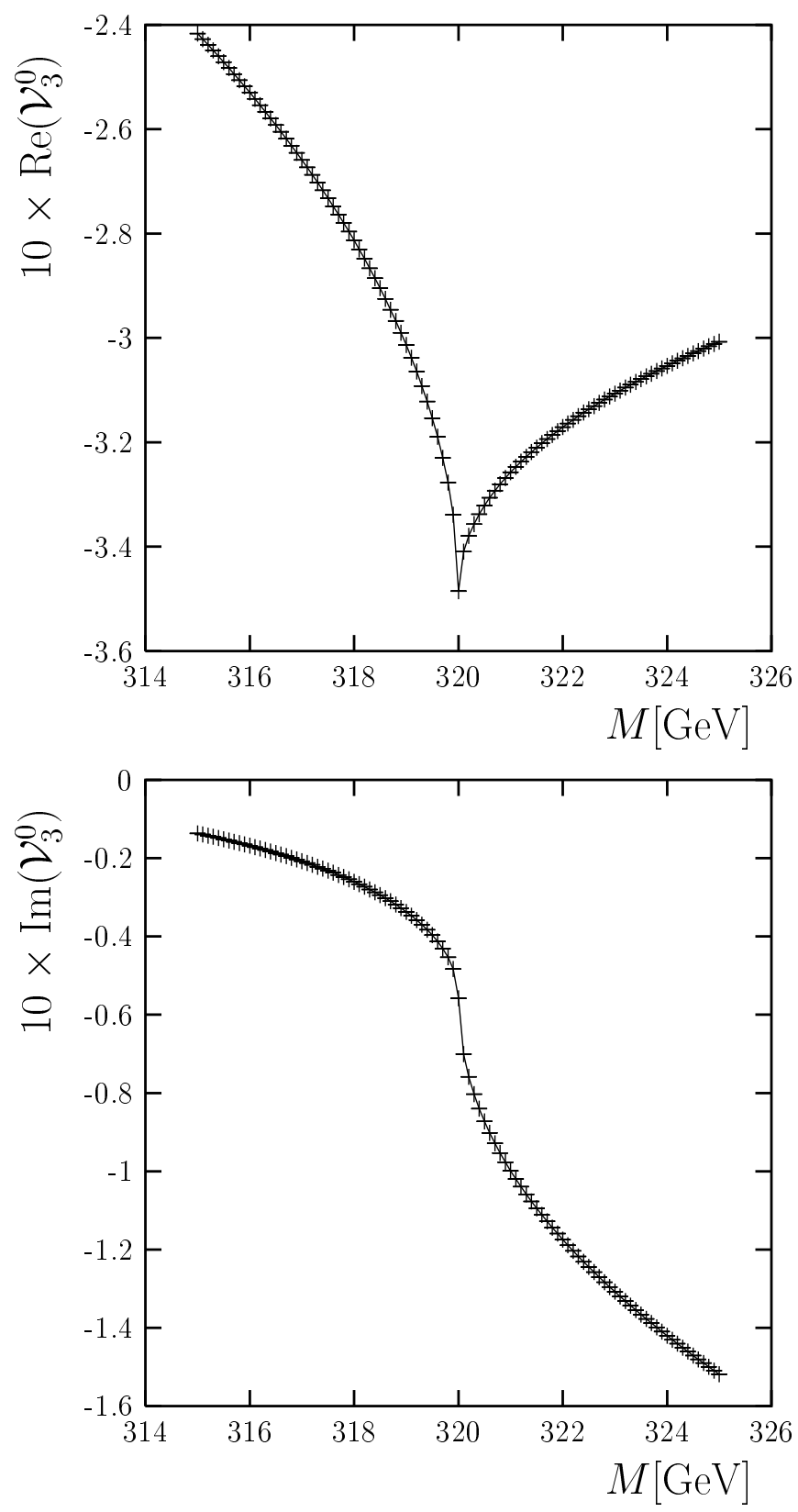

Figure 11: Real part (top) and imaginary part (bottom) of the integral $\mathcal{V}_{3}^{0}$ in Eq. (108) as a function of the decay mass $M=\sqrt{p^{2}}$ close to $M=320 \mathrm{GeV}$. The values for the masses and outgoing momenta are taken from Eq. (101). 

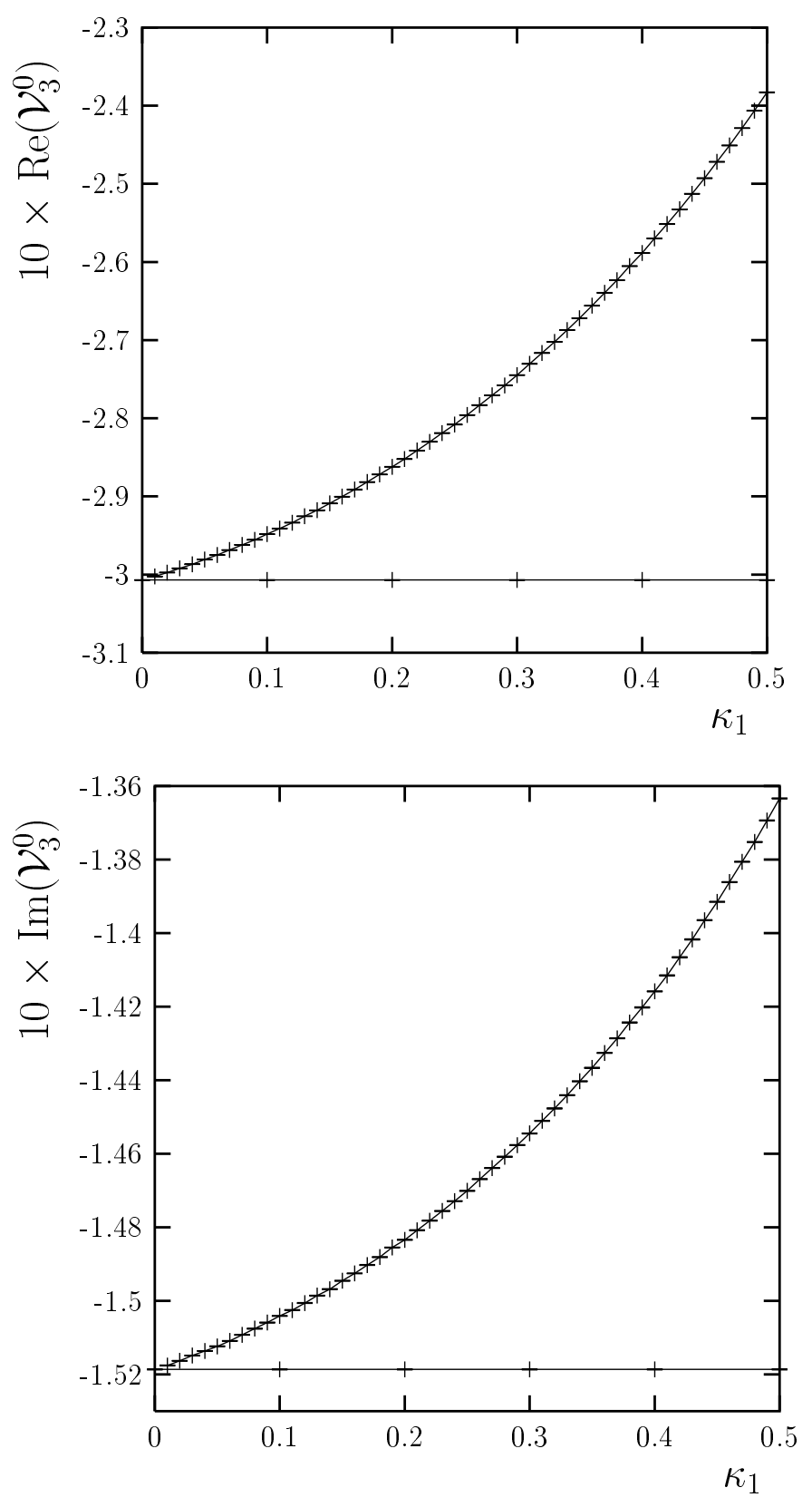

Figure 12: Real part (top) and imaginary part (bottom) of the integral $\mathcal{V}_{3}^{0}$ in Eq. (108) as a function of the parameters $\kappa_{1}$ and $\kappa_{2}=0.6 \cdot \kappa_{1}$ for the decay mass value $M=\sqrt{p^{2}}=$ $325 \mathrm{GeV}$. Shown are the results for the numerical calculation (curve) and for the semianalytical calculation with $\kappa_{2}=\kappa_{1}=0$ (horizontal straight line), both obtained by using VEGAS. 


\begin{tabular}{|r|lll|lll|}
\hline degree & real part & $\sigma_{\operatorname{Re}} \chi_{\mathrm{Re}}^{2} /(n-1)$ & imag. part & $\sigma_{\operatorname{Im}} \chi_{\operatorname{Im}}^{2} /(n-1)$ \\
\hline 3 & $-3.00814 \cdot 10^{-1}$ & $1.2 \cdot 10^{-5}$ & $4.2 \cdot 10^{0}$ & $-1.519135 \cdot 10^{-1}$ & $5.2 \cdot 10^{-6}$ & $3.6 \cdot 10^{0}$ \\
4 & $-3.00692 \cdot 10^{-1}$ & $1.5 \cdot 10^{-5}$ & $8.9 \cdot 10^{-1}$ & $-1.518660 \cdot 10^{-1}$ & $6.8 \cdot 10^{-6}$ & $1.2 \cdot 10^{0}$ \\
\hline $\mathrm{x}$ & $-3.00689 \cdot 10^{-1}$ & $1.7 \cdot 10^{-5}$ & & $-1.51865 \cdot 10^{-1}$ & $1.4 \cdot 10^{-5}$ & \\
\hline
\end{tabular}

Table 5: Extrapolation of the contributions for $\mathcal{V}_{3}^{0}$ using VEGAS

\begin{tabular}{|r|ll|llr|}
\hline degree & real part & $\sigma_{\operatorname{Re}} \chi_{\mathrm{Re}}^{2} /(n-1)$ & imag. part & $\sigma_{\operatorname{Im}} \chi_{\operatorname{Im}}^{2} /(n-1)$ \\
\hline $\mathrm{x}$ & $-3.0083 \cdot 10^{-1}$ & $2.9 \cdot 10^{-4}$ & $-1.5196 \cdot 10^{-1}$ & $1.4 \cdot 10^{-4}$ \\
\hline
\end{tabular}

Table 6: Extrapolation of the contributions for $\mathcal{V}_{3}^{0}$ using Divonne

\subsection{Combined integration}

After having performed these tests, the semi-analytical evaluation of the partial results from the different master integrals appears to be feasible. This might be done by performing the numerical integrations separately for each master integral and afterwards combining the results. However, it turns out that the more stable method is to first perform the analytical integrations separately and then to do the numerical integration for the sum of all basic integrands relevant for a given process. This is especially true for squared momenta on the threshold or close to it. As an example we use

$$
\mathcal{T}_{\text {ex }}=\int \frac{(k l)\left(p_{1} k\right)\left(p_{2} k+p_{2} l\right)}{P_{1} P_{2} P_{3} P_{4} P_{5} P_{6}} d^{D} k d^{D} l .
$$

After the tensor reduction explained in Sec. 3, we are left with the original planar topology and the two rotated reduced planar topologies, both with different powers of $\left(k_{0} \mp k_{1}\right)$ and $\left(l_{0} \mp l_{1}\right)$, and diagrams of simpler topologies. The master integrals for the non-reduced and rotated reduced planar topologies can be calculated by applying the subtractions explained in Sec. 4. For the convergent part the limit $\kappa_{i}, \lambda_{i} \rightarrow 0$ can be performed. All integrations up to the last two can be done analytically. The combination of the different UV-finite contributions with planar and rotated reduced planar topology at this point is called $\mathcal{V}_{\text {ex }}$.

As explained before, there are two possibilities to proceed. We can combine all analytical results to a single expression and integrate this expression numerically (combined integration), or we can perform the numerical integration of the analytical expression for each of the master integrals separately and add up the results afterwards (separated integration). We will perform both calculations in the following to compare them.

For the physical masses and outgoing momenta we again use the values in Eq. (101). 
The square of the decay mass $M=\sqrt{p^{2}}=500 \mathrm{GeV}$ is chosen to be equal to the two-particle threshold $p^{2}=\left(m_{5}+m_{6}\right)^{2}$. For the subtraction masses we take values with an offset $m_{0}$ which in this example runs from $0 \mathrm{GeV}$ to $200 \mathrm{GeV},{ }^{5}$

$$
\begin{array}{ll}
m_{1,1}=5 \mathrm{GeV}+m_{0} & m_{1,2}=25 \mathrm{GeV}+m_{0} \\
m_{2,1}=15 \mathrm{GeV}+m_{0} & m_{2,2}=35 \mathrm{GeV}+m_{0} .
\end{array}
$$

Subtraction terms and corresponding subtraction masses for the second loop momentum $l$ are not necessary, since in this particular case the contributing planar and rotated reduced planar topologies do not contain subdivergences in $l$. The results are shown in Fig. 13. The left hand side of Fig. 13] shows real and imaginary parts for the separated integration, the right hand side shows real and imaginary parts for the combined integration. It is obvious that the combined integration is much more stable.

This result is easy to understand. Assuming that the relative variances are the same for all $n$ partial integrals and for the complete integral, we can use the same number of intersection points in the Monte Carlo integration for each seperate integral and for the complete integral. In this case, the standard deviation of the seperately integrated and summed integrals is bigger by a factor of $\sqrt{n}$ than the standard deviation of the complete integral 63, 64].

\section{Conclusions}

Using the parallel/orthogonal space method, we have elaborated on a new method to calculate the planar and rotated reduced planar ladder two-loop three-point diagrams in the general massive case. Our method does not depend on a specific physical decay process but is suitable to calculate all planar two-loop vertex diagrams which may arise in the Standard Model of electroweak interactions using Feynman gauge.

We have presented a tensor reduction which reduces the mentioned topologies with arbitrary tensor structure to a set of master integrals with strongly restricted, well-defined numerator structure. We have developed and implemented an algorithm for the semianalytical calculation of the UV-finite part of those master integrals which still have the mentioned topologies after tensor reduction. While six of the eight integrations are done analytically, for the remaining two integrations we used different numerical methods.

\footnotetext{
${ }^{5}$ Note that $\mathcal{V}_{\text {ex }}$ still does not need to be independent of the subtraction masses because only in the sum with the finite parts of the subtraction terms this dependence will vanish.
} 

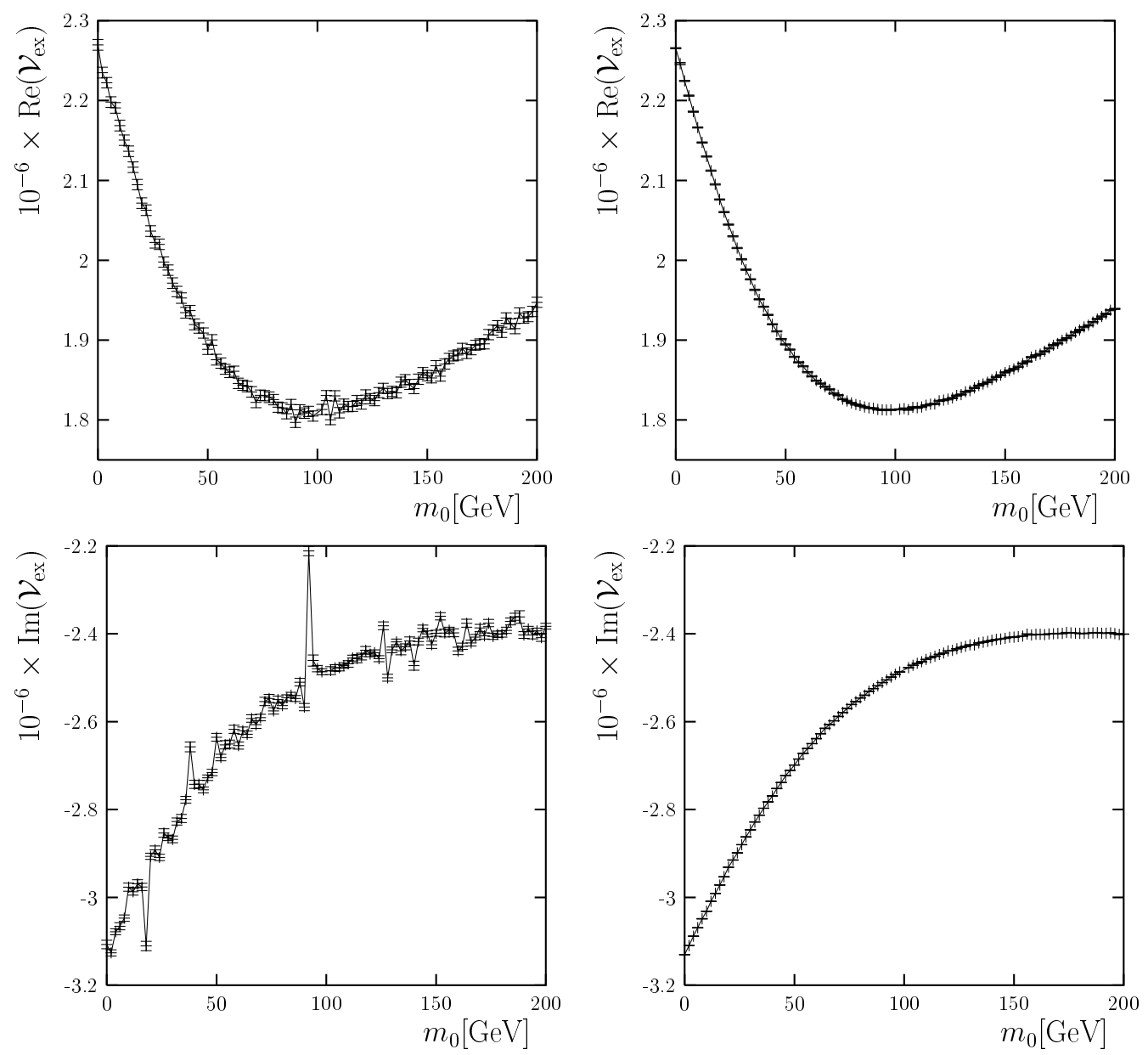

Figure 13: Real part (top) and imaginary part (bottom) of the integral $\mathcal{V}_{\text {ex }}$ as a function of the mass parameter $m_{0}$ of the subtraction masses for the decay mass value on the twoparticle threshold at $M=\sqrt{p^{2}}=500 \mathrm{GeV}$. The integral $\mathcal{V}_{\text {ex }}$ is the sum of the UV-finite parts of the master integrals corresponding to $\mathcal{T}_{\text {ex }}$ in Eq. (109) which after tensor reduction keep the planar and rotated reduced planar topology. Shown are the results for separated (left hand side) and combined numerical integration (right hand side) with VEGAS. 
The integration of the UV-finite part of the important master integrals was checked exhaustively in the limit of vanishing subtraction parameters by comparing the semianalytical calculation with the results of a numerical integration. As the final step of the semi-analytical calculation, the numerical integration turns out to be stable, i.e. one obtains the same result as for the above mentioned limit. This is valid especially in the case where the numerical integration is not done for each master integral separately but for the sum of all analytical results necessary for the given process.

For the remaining master integrals and subtraction terms with simpler topologies there exist other methods. In part, these methods are already implemented in xloops. Therefore, in writing links to existing codes, these cases can easily be implemented into our calculations. The still missing topologies are the two-loop three-point topologies containing a two-point subloop. These parts still have to be implemented [24, 25]. After having done this, we will be able to evaluate semi-analytically all IR-finite NNLO corrections containing planar two-loop vertex topologies as they arise in the Standard Model.

Finally, because the calculation of the scalar integrals for the non-planar two-loop vertex function [54] is quite similar to the calculation of the scalar integrals for the planar two-loop vertex function [34, our algorithm is expected to be easily extendable to the non-planar two-loop vertex topology. Having extended our algorithm in this direction, we are then able to calculate all NNLO vertex corrections as they arise in the Standard Model.

As far as possible, the computer codes written in the course of this paper are developed and implemented for most general decay processes. Because of this, we plan to include our calculations in the xloops-project in the near future.

\section{Acknowledgements}

We thank H.S. Do, J.G. Körner, A.v. Hameren, M. Rogal, K. Schilcher, C. Schwinn, H. Spiesberger, and E. Tuiran for helpful discussions. M.K. wants to thank C. Bauer and J. Vollinga for computer-technical support. S.G. acknowledges support by the DFG as a guest scientist in Mainz, by the Estonian target financed project No. 0182647s04, and by the Estonian Science Foundation under grant No. 6216. M.K. was supported in parts by the BMBF and by the Graduiertenkolleg "Eichtheorien - experimentelle Tests und theoretische Grundlagen", Mainz University. 


\section{A Integral basis}

In this appendix we list the analytical results for the basic integrals in Eq. (90) in terms of the parameters $r_{s}, r_{t}, r_{0}, s_{0}$, and $t_{0}$. In order to calculate these integrals, we introduce cutoffs $\Lambda_{s}$ and $\Lambda_{t}$. The results depending on these cutoffs are extremely long and contain products of two logarithms as well as dilogarithms [62]. However, one can show that these divergences cancel in the sums in Eqs. (67) and (88). In order to make the reader familiar with these features, we explain the calculation for the simplest example.

\section{A.1 The integral $\mathcal{F}_{0,0}^{s t}$}

The regularized integral is given by

$$
\begin{aligned}
\mathcal{F}_{0,0}^{s t} & =\int_{0}^{\Lambda_{t}} d t \int_{0}^{\Lambda_{s}} d s \frac{1}{\sqrt[\star]{\left(r_{0}-r_{s} s-r_{t}+i \eta\right)^{2}-4 s t}} \\
= & -\frac{\Lambda_{t}}{r_{s}} \ln \left|\frac{r_{s} r_{0}+\left(2-r_{s} r_{t}\right) \Lambda_{t}-r_{s}^{2} \Lambda_{s}-r_{s} \Lambda}{2\left(r_{s} r_{0}+\left(1-r_{s} r_{t}\right) \Lambda_{t}\right)}\right|-\frac{\Lambda_{s}}{r_{t}} \ln \left|\frac{r_{t} r_{0}+\left(2-r_{s} r_{t}\right) \Lambda_{s}-r_{t}^{2} \Lambda_{t}-r_{t} \Lambda}{2\left(r_{t} r_{0}+\left(1-r_{s} r_{t}\right) \Lambda_{s}\right)}\right| \\
& +\frac{r_{0}}{1-r_{s} r_{t}}\left(\ln \left|\Lambda_{t}+\frac{r_{s} r_{0}}{1-r_{s} r_{t}}\right|+\ln \left|\Lambda_{s}+\frac{r_{t} r_{0}}{1-r_{s} r_{t}}\right|+i \pi \theta\left(r_{0}\right)\right. \\
& \left.-\ln \left|r_{0}\left(1+r_{s} r_{t}\right)+\left(1-r_{s} r_{t}\right)\left(r_{s} \Lambda_{s}+r_{t} \Lambda_{t}+\Lambda\right)\right|-\ln \left|\frac{r_{0}}{2\left(1-r_{s} r_{t}\right)^{2}}\right|\right)
\end{aligned}
$$

where

$$
\Lambda:=\sqrt{\left(r_{0}-r_{s} \Lambda_{s}-r_{t} \Lambda_{t}\right)^{2}-4 \Lambda_{s} \Lambda_{t}}
$$

What to do with the singularity? As a first step we can separate the divergent part which depends on $\Lambda_{s}, \Lambda_{t}$ and $\Lambda$ from the convergent part. But how does the divergent part cancel? If we look at Eq. (88) where $\mathcal{F}_{0,0}^{s t}$ appears, we see that it actually appears as part of a sum. If we concentrate on the summation over $n$ and consider how $\mathcal{N}_{1, i, a}^{k}$ and $\mathcal{N}_{2, i, a}^{k}$ are defined in Eqs. (53) and (54), and if we take into account that $\left.P_{2, j}\right|_{P_{1, j}}=\xi_{2, j}-\xi_{1, j}=-\left.P_{1, j}\right|_{P_{2, j}}$, we see that $\mathcal{N}_{1, i, a}^{k}=-\mathcal{N}_{2, i, a}^{k}$. The same is valid for $\mathcal{N}_{x, j, b}^{l}$ and $\mathcal{N}_{6, j, b}^{l}$, i.e. $\mathcal{N}_{x, j, b}^{l}=-\mathcal{N}_{6, j, b}^{l}$. Therefore, an expression which is of relevance for the subtracted integrals is the sum

$$
\sum_{n=1}^{2} \sum_{m=x, 6}(-1)^{n+m} \mathcal{F}_{0,0}^{s t}\left(r_{s}, r_{t}, r_{n, i, m, j}\right) \text {. }
$$


In replacing $\Lambda_{s}$ by $\Lambda_{s}^{0} / \epsilon_{\Lambda}$ and $\Lambda_{t}$ by $\Lambda_{t}^{0} / \epsilon_{\Lambda}$ with $\epsilon_{\Lambda} \ll 1$ with arbitrary but positive and fixed values $\Lambda_{s}^{0}$ and $\Lambda_{t}^{0}$ we indeed can show that

$$
\sum_{n=1}^{2} \sum_{m=x, 6}(-1)^{n+m} \mathcal{F}_{0,0}^{s t, \operatorname{div}}\left(r_{s}, r_{t}, r_{n, i, m, j}\right)=O\left(\epsilon_{\Lambda}\right) .
$$

For $\epsilon_{\Lambda} \rightarrow 0$, therefore, the divergent part will cancel in the difference. We only have to consider the convergent part

$$
\mathcal{F}_{0,0}^{s t, \text { conv }}=\frac{r_{0}}{1-r_{s} r_{t}}\left(i \pi \theta\left(r_{0}\right)-\ln \left|\frac{r_{0}}{2\left(1-r_{s} r_{t}\right)^{2}}\right|\right) .
$$

Finally, we see that

$$
\sum_{n=1}^{2} \sum_{m=x, 6}(-1)^{n+m} r_{n, i, m, j} \rightarrow \frac{ \pm 2}{\theta_{0}} \sum_{m=x, 6}\left(\xi_{1, i}-\xi_{2, i}\right)=0
$$

(cf. Eq. (91) including remarks given there). Therefore, we can skip all terms which are linear in $r_{0}$. The result which we have to implement is

$$
\mathcal{F}_{0,0}^{s t, \text { impl }}=\frac{r_{0}}{1-r_{s} r_{t}}\left(i \pi \theta\left(r_{0}\right)-\ln \left|r_{0}\right|\right)
$$

In the following we will only give results which are to be implemented. For the integrals with one index we will show only results for $\mathcal{F}_{\alpha}^{s}$. Results for $\mathcal{F}_{\beta}^{t}$ can easily be obtained by replacing $s_{0} \leftrightarrow t_{0}$ and $r_{s} \leftrightarrow r_{t}$.

The integral $\mathcal{F}_{0,0}^{s t}$ is used for illustrative reasons only. It does not appear in calculations because the integration region vanishes. The same is valid for the integrals $\mathcal{F}_{i, j}^{s t}$. Therefore, it turns out that the integrals $\mathcal{F}_{\alpha}^{s}$ and $\mathcal{F}_{\beta}^{t}$ are sufficient for calculations within the Standard Model of electroweak interactions using Feynman gauge.

\section{A.2 The integrals $\mathcal{F}_{\alpha}^{s}$}

$$
\begin{aligned}
r_{s} \mathcal{F}_{0}^{s, \text { impl }}= & -\operatorname{Re}\left(\operatorname{Li}_{2}\left(1+\frac{r_{s} r_{0}}{t_{0}\left(1-r_{s} r_{t}\right)}\right)\right) \\
& -i \pi\left\{\theta\left(r_{0}\right) \ln \left|1+\frac{r_{s} r_{0}}{t_{0}\left(1-r_{s} r_{t}\right)}\right|-\theta\left(t_{0}\right) \ln \left|t_{0}+\frac{r_{s} r_{0}}{1-r_{s} r_{t}}\right|\right\} \\
r_{s}^{3} \mathcal{F}_{1}^{s, \text { impl }}= & -\left(2-r_{s} r_{t}\right)\left\{\frac{r_{s} r_{0}}{1-r_{s} r_{t}} \ln \left|r_{0}\right|+\left(t_{0}+\frac{r_{s} r_{0}}{2-r_{s} r_{t}}\right) \operatorname{Re}\left(\operatorname{Li}\left(1+\frac{r_{s} r_{0}}{t_{0}\left(1-r_{s} r_{t}\right)}\right)\right)\right\} \\
& +i \pi\left\{\left(2-r_{s} r_{t}\right) \theta\left(r_{0}\right)\left(\frac{r_{s} r_{0}}{1-r_{s} r_{t}}-\left(t_{0}+\frac{r_{s} r_{0}}{2-r_{s} r_{t}}\right) \ln \left|1+\frac{r_{s} r_{0}}{t_{0}\left(1-r_{s} r_{t}\right)}\right|\right)\right.
\end{aligned}
$$




$$
\begin{aligned}
& \left.+\left(\left(2-r_{s} r_{t}\right) t_{0}+r_{s} r_{0}\right) \theta\left(t_{0}\right) \ln \left|t_{0}+\frac{r_{s} r_{0}}{1-r_{s} r_{t}}\right|\right\} \\
r_{s}^{5} \mathcal{F}_{2}^{s, \text { impl }}= & \left\{\frac{1}{2}\left(\frac{r_{s} r_{0}}{1-r_{s} r_{t}}\right)^{2}\left(6-6 r_{s} r_{t}+r_{s}^{2} r_{t}^{2}\right)\right. \\
& \left.-\frac{r_{s} r_{0}}{1-r_{s} r_{t}}\left(\left(6-6 r_{s} r_{t}+r_{s}^{2} r_{t}^{2}\right) t_{0}+2\left(3-r_{s} r_{t}\right) r_{s} r_{0}\right)\right\} \ln \left|r_{0}\right| \\
- & \left(\left(6-6 r_{s} r_{t}+r_{s}^{2} r_{t}^{2}\right) t_{0}^{2}+2\left(3-r_{s} r_{t}\right) r_{s} r_{0} t_{0}+r_{s}^{2} r_{0}^{2}\right) \operatorname{Re}\left(\operatorname{Li}\left(1+\frac{r_{s} r_{0}}{t_{0}\left(1-r_{s} r_{t}\right)}\right)\right) \\
-i & \left\{\theta ( r _ { 0 } ) \left(\frac{1}{2}\left(\frac{r_{s} r_{0}}{1-r_{s} r_{t}}\right)^{2}\left(6-6 r_{s} r_{t}+r_{s}^{2} r_{t}^{2}\right)\right.\right. \\
& \quad-\frac{r_{s} r_{0}}{1-r_{s} r_{t}}\left(\left(6-6 r_{s} r_{t}+r_{s}^{2} r_{t}^{2}\right) t_{0}+2\left(3-r_{s} r_{t}\right) r_{s} r_{0}\right) \\
& \left.+\left(\left(6-6 r_{s} r_{t}+r_{s}^{2} r_{t}^{2}\right) t_{0}^{2}+2\left(3-r_{s} r_{t}\right) r_{s} r_{0} t_{0}+r_{s}^{2} r_{0}^{2}\right) \ln \left|1+\frac{r_{s} r_{0}}{t_{0}\left(1-r_{s} r_{t}\right)}\right|\right) \\
- & \left.\theta\left(t_{0}\right)\left(\left(6-6 r_{s} r_{t}+r_{s}^{2} r_{t}^{2}\right) t_{0}^{2}+2\left(3-r_{s} r_{t}\right) r_{s} r_{0} t_{0}+r_{s}^{2} r_{0}^{2}\right) \ln \left|t_{0}+\frac{r_{s} r_{0}}{1-r_{s} r_{t}}\right|\right\}
\end{aligned}
$$

\section{References}

[1] W. Hollik and G. Duckeck, "Electroweak Precision Tests at LEP", Springer 2000

[2] D.Y. Bardin, J. Phys. G29 (2003) 75

[3] W. Hollik et al., Acta Phys. Polon. B35 (2004) 2533

[4] [LEP Collaborations], "Precision electroweak measurements on the $Z$ resonance", Report No. CERN-PH-EP-2005-041, arXiv:hep-ex/0509008, submitted to Physics Reports

[5] J. A. Aguilar-Saavedra et al. [ECFA/DESY LC Physics Working Group], "TESLA Technical Design Report Part III: Physics at an $e^{+} e^{-}$Linear Collider", Report No. DESY-TESLA-2001-23 arXiv:hep-ph/0106315

[6] S. Heinemeyer and G. Weiglein, "Electroweak precision tests with GigaZ", published in Batavia 2000, Physics and experiments with future linear $e^{+} e^{-}$colliders, pp. 511-514, Report No. LC-TH-2001-001 arXiv:hep-ph/0012364 
[7] G. Weiglein, Eur. Phys. J. C33 (2004) S630

[8] G. Weiglein et al. [LHC/LC Study Group], "Physics interplay of the LHC and the ILC", Report No. SLAC-PUB-10764 arXiv:hep-ph/0410364

[9] S. Heinemeyer, "Loop calculations: Summary",

Report no. CERN-PH-TH-2004-159 arXiv:hep-ph/0408269

[10] D. Kreimer, Phys. Lett. B292 (1992) 341

[11] N.I. Usyukina and A.I. Davydychev, Phys. Lett. B348 (1995) 503

[12] A.I. Davydychev and J.B. Tausk, Nucl. Phys. B465 (1996) 507

[13] J. Fujimoto, Y. Shimizu, K. Kato and T. Kaneko, Int. J. Mod. Phys. C6 (1995) 525

[14] A.A. Pivovarov, Phys. Atom. Nucl. 63 (2000) 1646 [Yad. Fiz. 63N9 (2000) 1734]

[15] K.G. Chetyrkin, R. Harlander, J.H. Kühn and M. Steinhauser, Nucl. Instrum. Meth. A389 (1997) 354

[16] S. Groote, J.G. Körner and A.A. Pivovarov, Phys. Lett. B443 (1998) 269; Nucl. Phys. B542 (1999) 515; Eur. Phys. J. C11 (1999) 279; Phys. Rev. D60 (1999) 061701; Phys. Rev. D61 (2000) 071501; Phys. Rev. D65 (2002) 036001; Eur. Phys. J. C36 (2004) 471; "On the evaluation of a certain class of Feynman diagrams in $x$-space: Sunrise-type topologies at any loop order", Report No. MZ-TH-05-08 arXiv:hep-ph/0506286; S. Groote and A.A. Pivovarov, Nucl. Phys. B580 (2000) 459

[17] A. Ghinculov and Y.P. Yao, Nucl. Phys. B516 (1998) 385; Phys. Rev. D63 (2001) 054510

[18] A.I. Davydychev and V.A. Smirnov, Nucl. Instrum. Meth. A502 (2003) 621

[19] U. Aglietti and R. Bonciani, Nucl. Phys. B668 (2003) 3; B698 (2004) 277

[20] W. Bernreuther, R. Bonciani, T. Gehrmann, R. Heinesch, T. Leineweber, P. Mastrolia and E. Remiddi, Nucl. Phys. B706 (2005) 245; B712 (2005) 229 
[21] R. Bonciani, P. Mastrolia and E. Remiddi, Nucl. Phys. B661 (2003) 289 [Erratum B702 (2004) 359]; B676 (2004) 399; B690 (2004) 138;

R. Bonciani, A. Ferroglia, P. Mastrolia, E. Remiddi and J.J. van der Bij, Nucl. Phys. B701 (2004) 121

[22] M. Caffo, H. Czyż and E. Remiddi, Nucl. Phys. Proc. Suppl. 116 (2003) 422

[23] P. Mastrolia and E. Remiddi, Nucl. Phys. Proc. Suppl. 116 (2003) 412

[24] S. Bauberger, F.A. Berends, M. Böhm and M. Buza, Nucl. Phys. B434 (1995) 383;

S. Bauberger, M. Böhm, G. Weiglein, F.A. Berends and M. Buza, Nucl. Phys. Proc. Suppl. 37B (1994) 95

[25] P. Post, Ph.D. thesis, Mainz University, 1997

[26] P. Post and J.B. Tausk, Mod. Phys. Lett. A11 (1996) 2115

[27] H.S. Do, Ph.D. thesis, Mainz University, 2003

[28] G. Passarino, Nucl. Phys. B619 (2001) 257

[29] A. Ferroglia, M. Passera, G. Passarino and S. Uccirati, Nucl. Phys. B680 (2004) 199

[30] S. Actis, A. Ferroglia, G. Passarino, M. Passera and S. Uccirati, Nucl. Phys. B703 (2004) 3

[31] W. Hollik, U. Meier and S. Uccirati, "The effective electroweak mixing angle $\sin ^{2}\left(\theta_{\text {eff }}\right)$ with two-loop fermionic contributions", Report No. MPP-2005-77 arXiv:hep-ph/0507158

[32] M. Awramik, M. Czakon, A. Freitas and G. Weiglein, Phys. Rev. Lett. 93 (2004) 201805; Nucl. Phys. Proc. Suppl. 135 (2004) 119

[33] D. Kreimer, Phys. Atom. Nucl. 56 (1993) 1546

[34] A. Czarnecki, U. Kilian and D. Kreimer, Nucl. Phys. B433 (1995) 259

[35] A. Frink, U. Kilian and D. Kreimer, Nucl. Phys. B488 (1997) 426 
[36] D. Kreimer, Mod. Phys. Lett. A9 (1994) 1105

[37] A.V. Kotikov, Phys. Lett. B259 (1991) 314

[38] J. Fleischer, A.V. Kotikov and O.L. Veretin, Phys. Lett. B417 (1998) 163; Nucl. Phys. B547 (1999) 343

[39] V.A. Smirnov and E.R. Rakhmetov, Theor. Math. Phys. 120 (1999) 870 [Teor. Mat. Fiz. 120 (1999) 64]

[40] T.G. Birthwright, E.W.N. Glover and P. Marquard, JHEP 0409 (2004) 042

[41] J. Fleischer, V.A. Smirnov, A. Frink, J.G. Körner, D. Kreimer, K. Schilcher and J.B. Tausk, Eur. Phys. J. C2 (1998) 747

[42] A. Frink, Ph.D. thesis, Mainz University, 2000

[43] G.P. Lepage, "Vegas: An Adaptive Multidimensional Integration Program", Report No. CLNS-80/447

[44] T. Ohl, Comput. Phys. Commun. 120 (1999) 13

[45] T. Hahn, "CUBA: A library for multidimensional numerical integration", Report No. MPP-2004-40 arXiv:hep-ph/0404043

[46] D. Kreimer, Nucl. Instrum. Meth. A389 (1997) 323

[47] L. Brücher, Nucl. Instrum. Meth. A389 (1997) 327

[48] J. Franzkowski, Nucl. Instrum. Meth. A389 (1997) 333

[49] A. Frink, J.G. Körner and J.B. Tausk, "Massive two-loop integrals and Higgs physics", published in the Proceedings of the Joint Particle Physics Meeting, Ouranoupolis, Greece, May 1997, pp. 175-200

[50] C. Bauer and H.S. Do, Comput. Phys. Commun. 144 (2002) 154

[51] C. Bauer, A. Frink and R. Kreckel, Report No. MZ-TH/00-17 arXiv:cs.sc/0004015

[52] D. Kreimer, Z. Phys. C54 (1992) 667 
[53] D. Kreimer, Int. J. Mod. Phys. A8 (1993) 1797

[54] A. Frink, Diploma thesis, Mainz University, 1996

[55] G. Passarino and M.J.G. Veltman, Nucl. Phys. B160 (1979) 151

[56] A. Andonov et al., "SANCscope - v.0.41", arXiv:hep-ph/0411186

[57] R. Harlander and M. Steinhauser, Prog. Part. Nucl. Phys. 43 (1999) 167

[58] U. Kilian, Ph.D. thesis, Mainz University, 1996

[59] L.D. Landau, Nucl. Phys. 13 (1959) 181

[60] M. Böhm, A. Denner, H. Joos, "Gauge Theory of the Strong and Electroweak Interaction", Teubner, 2001

[61] R.E. Cutkosky, J. Math. Phys. 1 (1960) 429

[62] M.M. Knodel, Ph.D. thesis, Mainz University, 2005

[63] R.H. Kleiss and A. Lazopoulos, "Error in Monte Carlo, quasi-error in quasi-Monte Carlo", arXiv:hep-ph/0504085

[64] A.F.W. van Hameren, Ph.D. thesis, Nijmegen University, 2001 arXiv:hep-ph/0101094

[65] S. Eidelman et al. [Particle Data Group], Phys. Lett. B592 (2004) 1 